\title{
The Wild Emmer Wheat Gene PmG16, Conferring Resistance to Powdery Mildew, is an Orthologue of Pm60 from Triticum Urartu
}

\section{Yinghui Li}

University of Haifa

\section{Zhen-Zhen Wei}

University of Haifa

Andrii Fatiukha

University of Haifa

\section{Samidha Jaiwar}

University of Haifa

Hanchao Wang

University of Haifa

\section{Samiha Hasan}

University of Haifa

Zhiyong Liu

Chinese Academy of Sciences

Hanan Sela

University of Haifa

Tamar Krugman

University of Haifa

Tzion Fahima ( $\square$ fahima@research.haifa.ac.il)

University of Haifa https://orcid.org/0000-0001-5555-0040

\section{Original Article}

Keywords: Blumeria graminis, wild emmer wheat, molecular marker, PmG16, Pm60

Posted Date: February 3rd, 2021

DOI: https://doi.org/10.21203/rs.3.rs-166805/v1

License: (c) (i) This work is licensed under a Creative Commons Attribution 4.0 International License.

Read Full License 


\section{Abstract}

Wild emmer wheat (WEW), the tetraploid progenitor of durum and bread wheat, is a valuable genetic resource for resistance to powdery mildew fungal disease caused by Blumeria graminis f. sp. tritici (Bgt). PmG16 gene, derived from WEW, confers high resistance to most tested Bgt isolates. We mapped PmG16 to a $1.4 \mathrm{cM}$ interval between the flanking markers uhw386 and uhw390 on Chromosome 7AL. Based on gene annotation of WEW reference genome Zavitan_V1, 34 predicted genes were identified within the $\sim 3.48 \mathrm{Mb}$ target region. Six genes were annotated as associated with disease resistance, of which TRIDC7AG077150.1 was found to be highly similar to Pm60, previously cloned from Triticum urartu and residing in the same syntenic region. A functional molecular marker (FMM) for Pm60 (M-Pm60-S1) cosegregated with PmG16, suggesting that WEW PmG16 is probably an orthologue of Pm60 from Triticum urartu (designated here as TdPm60). Sequence alignment identified only eight SNPs that differentiate between TdPm60 and TuPm60. Furthermore, our results suggest that other WEW powdery mildew resistance genes MIIW172 and MIIW72, that also mapped to the same region of Chromosome 7AL, might be identical or allelic to TdPm60. Screening of 230 WEW accessions with Pm60 specific markers, 58 resistant accessions were identified from Southern Levant harboring the TdPm60 allele, while all the susceptible accessions showed no PCR amplifications. Deployment of TdPm60 is clearly more advantageous over TuPm60 since it can be rapidly introgressed by classical breeding approaches into bread wheat genetic background.

\section{Introduction}

Bread wheat (Triticum aestivum L., $2 \mathrm{n}=6 \mathrm{x}=42$, AABBDD) is one of the most important cereal crops, providing $20 \%$ of calories and proteins consumed by humans (Asif et al. 2014). Powdery mildew caused by the biotrophic fungus Blumeria graminis f. sp. tritici $(B g t)$, is one of the most destructive wheat diseases worldwide causing $5 \%-40 \%$ of yield losses (Singh et al. 2016). Deployment of effective powdery mildew resistance genes (Pm genes) is a sustainable and economical approach to reduce yield losses caused by this pathogen. The efforts towards this goal resulted in the mapping of more than 100 alleles in about 60 loci in wheat or in its wild relatives (Mclntosh et al. 2019). Among officially designated $P m$ genes (Pm1 to Pm68), 10 genes were cloned and characterized so far. Of them, $P m 3 / P m 8, P m 2, P m 21$, Pm60, Pm5e, Pm41 and Pm1a encode nucleotide-binding domain and leucine-rich repeat (NLR) immune receptors (Yahiaoui et al. 2004; Hurni et al. 2013; Sánchez-Martín et al. 2016; Xing et al. 2018; He et al. 2018; Zou et al. 2018; Xie et al. 2020; Li et al. 2020; Hewitt et al. 2020), and Pm24 encodes a tandem kinase protein (Lu et al. 2020). Besides, Pm38 (Lr34/ Yr18/Sr57/ Pm38 multi-resistance gene) encodes an ABC transporter (Krattinger et al. 2009) and Pm46 ( $\mathrm{rr67/Yr46/Sr55/Pm46} \mathrm{multi-resistance} \mathrm{gene)} \mathrm{encodes}$ a hexose transporter (Moore et al. 2015). The two latter non-NLR genes showed a broad-spectrum adult plant resistance to powdery mildew and rust diseases.

Wild emmer wheat (WEW), the tetraploid progenitor of cultivated wheat, is a valuable genetic resource for powdery mildew resistance breeding. Currently, more than $24 \mathrm{Pm}$ genes and one quantitative resistance loci have been identified and mapped in WEW. Seven of them (Pm16, Pm26, Pm30, Pm36, Pm41, Pm42 
and Pm64) have been officially designated as Pm resistance genes (Klymiuk et al. 2019; Mclntosh et al. 2019; Zhang et al. 2019). Other temporarily named $P m$ resistance genes, MIIW72, PmG16, MIWE18, MIIW172 and HSM1 were mapped in the same genomic region of Chromosome (Chr) 7AL of WEW (at the terminal bin 0.86-1.00 of 7AL). Besides, Pm1a from bread wheat (Neu et al. 2002), PmU (Pm60) from T. urartu (Qiu et al. 2005; Zhang et al. 2018) and PmTb7A.2 from T. boeoticum (Chhuneja et al. 2015) were also mapped to the same locus on the terminal part of Chr 7AL. The sequence of Pm1a which was cloned from T. aestivum acc. Axminster is quite dissimilar to Pm60, therefore, it is unlikely that they represent orthologous genes (Hewitt et al. 2020). These findings suggest that an $R$-gene cluster is present within this genomic region of cultivated and wild wheat species of different ploidy levels, conferring powdery mildew resistance (Liang et al. 2016; Zhang et al. 2018). Recently, a multi-genome comparisons showed huge differences in NLR content between wheat genotypes; Among 2,500 loci with NLR signatures identified in the 16 wheat cultivars, $31-34 \%$ of them are shared across all genomes, while the number of unique signatures ranged from 22 to 192 per cultivar (Walkowiak et al. 2020).

Pm60 which encodes a typical CC-NB-LRR type of NLR immune receptor which was cloned from Chr 7AL of $T$. urartu acc. PI428309. Large sequence differences were found between the respective Pm60 alleles in PI428309 and the reference genome of T. urartu acc. G1812 (Zou et al. 2018). Two additional functional $P m 60$ alleles which differ in the LRR regions were identified from different $T$. urartu accessions, Pm60a in PI428210 that includes a 240-nucleotide deletion and Pm60b in PI428215 that includes a 240-nucleotide insertion as compared with the Pm60 in PI428309 (Zou et al. 2018). Two functional PCR markers for Pm60 (M-Pm60-S1 and M-Pm60-S2), covering the LRR region were developed for diagnosis of these three Pm60 alleles by amplicon size, i.e. the M-Pm60-S1 amplify 591 bp (Pm60a), $831 \mathrm{bp}(P m 60)$, or $1071 \mathrm{bp} \mathrm{(Pm60b)}$, while no amplification product is obtained in susceptible $T$. urartu accessions (Zhao et al. 2020). Marker M-Pm60-S1 amplified one of the three functional alleles in 78\% (73 out of 93) of the tested resistant T. urartu accessions. Furthermore, 34.8\% (67 out of 227) T. urartu accessions harbor the Pm60 allele, and 2.64\% accessions harbor Pm60a or Pm60b alleles (6 out of 227 of each allele) indicating that the Pm60 locus is one of the major Bgt resistance loci prevalent among natural populations of T. urartu (Zhao et al. 2020).

The dominant gene PmG16 was identified and mapped using a mapping population derived from the powdery mildew resistant Israeli WEW accession G18-16 crossed with a susceptible $T$. durum cultivar Langdon (LDN). PmG16 conferred resistance to 34 out of 47 tested isolates from Israel and Switzerland and it was physically mapped on Chr 7AL (Ben-David et al. 2010). In the present study, we fine-mapped PmG16 and identified a candidate gene, which was highly similar to Pm60 and was mapped to the Pm60 syntenic locus on Chr 7AL of WEW. Sequence and functional marker analyses suggested that the candidate gene (named as TdPm60) from WEW G18-16 is an orthologous allele of TuPm60 with only eight SNPs differentiating between them. To study the geographical distribution of TdPm60, we screened a WEW collection of 230 accessions from the Fertile Crescent using a specific selection marker for Pm60. This screening revealed that 58 WEW accessions harboring the TdPm60 allele were highly resistant to $B g t$, and that most of them originated from the Southern Levant. Using marker-assisted backcrossing 
program, we have successfully introgressed PmG16 into the susceptible bread wheat cultivar Ruta and validated the improvement of resistance to $B g t$ in the progenies.

\section{Materials And Methods Plant material}

The powdery mildew resistant accession WEW G18-16 containing PmG16 originated from Gitit (32.10N, 35.39E) (Peleg et al. 2008; Ben-David et al. 2010). A recombinant inbred line ( $\left.F_{7} R I L\right)$ population of 147 lines, derived from the cross between WEW G18-16 and T. durum var. Langdon (LDN), was used for fine mapping of PmG16 (Peleg et al. 2008; Ben-David et al. 2010; Fatiukha et al. 2019). Single nucleotide polymorphism (SNP) genotyping was performed using the Illumina Infinium 15K Wheat platform, developed by TraitGenetics, Gatersleben, Germany (Muqaddasi et al. 2017), consisting of 12,905 SNPs selected from the wheat $90 \mathrm{~K}$ array (Wang et al. 2014). PmG16 was introgressed into Israeli modern bread wheat cultivar Ruta following marker-assisted backcross approach, with four backcross and one selfing generations $\left(\mathrm{BC}_{4} \mathrm{~F}_{2}\right)$. Bread wheat Chinese Spring, durum wheat Kronos and Svevo, WEW Zavitan and G305-3M, together with the 230 WEW accessions originating from across the Fertile Crescent were used for Pm60 orthologous locus analysis. Seeds of those wheat accessions were obtained from the Wild Cereals Gene Bank (ICGB) at the University of Haifa, Israel.

\section{Bgt inoculation and disease assessment}

Bgt isolates - six Bgt isolates used for phenotyping were collected from wild and domesticated wheat species from various agro-ecological habitats across Israel (Ben-David et al. 2010). Bgt isolate \#15 (Bgt \#15) collected from T. durum was used for histopathological characterization of the defense responses, phenotyping of segregating mapping populations, and screening for resistance in the WEW collection and introgression lines. Other isolates, including Bgt \#70, Bgt\#101 and Bgt \#107 that were collected from bread wheat ( $T$. aestivum), and Bgt \#103 that was collected from WEW, were used for resistance spectrum identification of 29 wheat accessions with different Pm60 alleles. Isolate Bgt \#SH was collected from WEW in the screening house (SH) of the University of Haifa.

Disease assessment - young plants at the two-leaf stage were tested for resistance to powdery mildew. Resistance results were recorded at 7 and $10 \mathrm{dpi}$. Incubation and disease assessment were as described by Hsam and Zeller (1997) and Xu et al. (2018) with slight modifications. In brief, the first leaf of each plant was excised into three segments and cultured in square petri dishes containing $8 \mathrm{~g} / \mathrm{L}$ agar with 50 $\mathrm{mg} / \mathrm{L}$ benzimidazole (Sigma-Aldrich, USA). Dishes were inoculated with fresh spores of powdery mildew in a inoculation tower, and were transferred to a climate chamber with $70 \%$ humidity and maintained in $22^{\circ} \mathrm{C} / 20^{\circ} \mathrm{C}$ under $12 \mathrm{~h}$ light $/ 12 \mathrm{~h}$ dark photoperiod, respectively. The reaction to $\mathrm{Bgt}$ was examined visually, and infection type (IT) was recorded in a scale of $0-4$ : 0 , no visible symptoms; 0 ; hypersensitive reaction; 1 , disease spot of less than $1 \mathrm{~mm}$ with a thin hypha layer and fewer spores; 2 , disease spots of less than $1 \mathrm{~mm}$ with thick hypha layer and more spores; 3 , scattered disease spots of greater than $1 \mathrm{~mm}$ 
with a thick hypha layer and more spores; and 4, continuous disease spots of greater than $1 \mathrm{~mm}$ with a thick hypha layer and more spores. Plants with IT of 0-2 were considered as resistant and plants with IT of 3-4 were considered as susceptible.

\section{Bgt -wheat interactions}

Bgt \#15 structures were observed in the inoculated leaves under a fluorescence microscope as described by Klymiuk et al. (2018) and Wei et al. (2020). Leaf segments were incubated at $37^{\circ} \mathrm{C}$ for 24 hours in 15 $\mathrm{ml}$ of $1 \mathrm{M} \mathrm{KOH}$, neutralized with $50 \mathrm{mM}$ Tris at pH 7.5, and stained with $1.0 \mathrm{ml}$ of $20 \mu \mathrm{g} / \mathrm{ml}$ wheat germ agglutinin (WGA) in $50 \mathrm{mM}$ Tris for 24 hours at $4^{\circ} \mathrm{C}$. Fluorescence microscopy was carried out using an inverted fluorescence microscope Leica DMi8 (Leica Microsystems, Germany), fitted with a filter cube for the FITC excitation range to observe the WGA-stained fungal structures.

Reactive oxygen species (ROS) accumulation and the cell death were evaluated in inoculated wheat leaves (2 dpi). Methods were applied according to Thordal-Christensen et al. (1997) and van Wees (2008) with minor modifications. For the ROS detection, wheat leaves placed in $1 \mathrm{mg} \mathrm{mL}^{-1} \mathrm{DAB}$ solution $(\mathrm{w} / \mathrm{v})$ with pH $3.8\left(15\right.$ mins in vacuum pressure at $-60 \mathrm{KPa}, 8$ hours at $\left.28^{\circ} \mathrm{C}\right)$, decoloring for 2 days in $96 \%$ ethanol, staining of Bgt structure with 0.6\% Coomassie Brilliant Blue G250 (Sigma, UK; 0.6g dissolved in $100 \mathrm{~mL}$ absolute ethyl alcohol) for 2 mines and washing with sterile water, then stored in $50 \%$ glycerol. For the cell death detection, wheat leaves were stained for $5 \mathrm{~min}$ in $0.25 \%$ Trypan blue solution (Biological Industries, Israel) with incubating in boiling water, decoloring in $2.5 \mathrm{~g} / \mathrm{mL}$ Chloral hydrate (Riedel-dehaën, Germany) for 2 days, then stored in $50 \%$ glycerol. ROS and cell death were examined under a microscope Zeiss Axio Imager M1 (Carl Zeiss, Germany).

\section{Development of molecular markers and PCR detection}

Polymorphic SNPs around the genomic region of PmG16 were detected by genotyping the 147 RILs (Table $\mathrm{S} 1$ ) of the mapping population by the $15 \mathrm{~K}$ wheat SNP array (Trait Genetics ${ }^{\circledR}$, Germany).

Chromosome specific SSR primers linked to PmG16 were designed using the BatchPrimer3 website tools (https://probes.pw.usda.gov/batchprimer3/), based on polymorphism found between the reference genomes of wild emmer wheat WEW Zavitan_v2.0 (Zhu et al. 2019) and durum wheat Svevo_v1.0 (Maccaferri et al. 2019). SNP-based Kompetitive Allele Specific PCR (KASP) primers were developed using PolyMarker (http://polymarker.tgac.ac.uk/) based on the specific SNPs at position 795 (C/T) in the alinement of different Pm60 homologs by using DNAMAN software (Fig. S5). Detailed information of all primers is presented in Table S2 and S3.

Wheat genomic DNA was extracted following the cetyltrimethylammonium bromide (CTAB) method. PCR mixture $(20 \mu \mathrm{L})$ contained $50-100 \mathrm{ng}$ of template DNA, $0.4 \mu \mathrm{M}$ of forward and reverse primers, $10 \mu \mathrm{L}$ of $2 \times$ Taq PCR MasterMix (Tiangen, China). PCR program for SSR amplification included denaturation at $94^{\circ} \mathrm{C}$ for 5 mins; followed by 35 cycles of $94^{\circ} \mathrm{C}$ for $1 \mathrm{~min}$, annealing at $57-61^{\circ} \mathrm{C}$ for $35 \mathrm{~s}$ (depending on the $\mathrm{Tm} \otimes$ of each primer pair), and $72^{\circ} \mathrm{C}$ for an appropriate time depending on the length of PCR products ( $1 \mathrm{~min} / \mathrm{kb}$ ), elongation at $72^{\circ} \mathrm{C}$ for 10 mins, and termination at $10^{\circ} \mathrm{C}$ in a Veriti ${ }^{\circledR} 96-$ Well Fast Thermal 
Cycler (Applied Biosystems, Singapore). PCR amplicons were visualized on 1.5-3\% agarose gels. KASP genotyping was performed in $5 \mu$ l containing $2.5 \mu$ KASP Master mix (LGC Genomics, USA), $0.4 \mu \mathrm{mol} \mathrm{L}^{-1}$ of each of three primers and $200 \mathrm{ng}$ of template DNA. KASP PCR amplifications were performed in a StepOnePlus ${ }^{\text {TM }}$ Real-Time PCR System (Applied Biosystem, USA) with the following amplification conditions: an initial step at $30^{\circ} \mathrm{C}$ for $1 \mathrm{~min}, 94^{\circ} \mathrm{C}$ for $15 \mathrm{mins}$ followed by 10 cycles of $94^{\circ} \mathrm{C}$ for $20 \mathrm{~s}, 65^{\circ} \mathrm{C}$ for $1 \mathrm{~min}$ (drop $0.6^{\circ} \mathrm{C}$ per cycle); and continue with 26 cycles of $94^{\circ} \mathrm{C}$ for $20 \mathrm{~s}, 55^{\circ} \mathrm{C}$ for 1 min; terminated after $30^{\circ} \mathrm{C}$ for $1 \mathrm{~min}$.

\section{Development of genetic and physical maps}

The genetic distances between the detected markers and PmG16 were calculated based on genotypic (15K SNP arrays and SSR markers) and phenotypic (IT to Bgt \#15) data of 147 RILs by using JoinMap 4.0 (Van Ooijen et al. 2006) (Table S1). The locations and specificity of probe sequences of SNP markers included in the $15 \mathrm{~K}$ array and SSR markers were identified by using a command-line tool BLAST + from NCBI (Camacho et al. 2009) against the sequences of Chr 7A of WEW Zavitan_v2.0 (Zhu et al. 2019), durum wheat Svevo_v1.0 (Maccaferri et al. 2019) and T. urartu G1812 (Ling et al. 2018). The genetic and physical maps of PmG16 genomic region in Chr 7AL were constructed by MapChart (Voorrips et al. 2002).

\section{Gene cloning, phylogenetic, and NLR protein structure analysis}

Based on the genetic and physical maps of $P m G 16$ we have identified candidate genes that are residing in WEW Zavitan_v2.0 reference genome sequence between the markers flanking PmG16. The sequences of candidate genes were viewed in the target genetic region of PmG16 and downloaded from GrainGenes Zavitan download page (https://wheat.pw.usda.gov/graingenes_downloads/Zavitan/). Because the WEW Zavitan_v2.0 does not contain gene annotation information, we used the annotations of these candidate genes from the WEW reference genome Zavitan_v1.0. Four PCR primer sets (Table S3) were developed based on the published 9492 bp-length nucleotide sequence around Pm60 locus in T. urartu (Tang et al. 2017) and used for cloning and sequencing of the Pm60 alleles in different WEW accessions. Sequences of PCR products were assembled and analyzed using DNAMAN software (Version 9). The Pm60 homologs in the phylogeny tree were identified from the GrainGenes and Ensembl Plants website, and analyzed using the Neighbor-Joining algorithm (aligning by MUSCLE) in the MEGA-X software (Kumar et al. 2018). To study the protein structure of $P m 60$ alleles and homologs, we used the NLR annotator (Steuernagel et al. 2020), which annotates NLR genes by motifs as described in Jupe et al. (2012).

\section{RNA Extraction and Quantitative Real-Time RT-PCR (qPCR)}

Total RNA was extracted from both Bgt \#15-treated and mock-treated (as control) G18-16 leaf segments collected at four different time points (12, 24, 36 and 48 hpi) using RNeasy Plant Mini Kit (Qiagen, Germany). cDNA was synthesized from total RNA using a qScript ${ }^{\text {TM }}$ cDNA Synthesis Kit (Quantabio, USA). 
Gene-specific primers of the TdPm60 and the housekeeping gene Actin were used for qPCR (Table S3). qPCR amplifications were performed in a volume of $10 \mu$ containing $5 \mu$ l of SYBR Green FastMix (Quantabio, USA), $250 \mathrm{nM}$ primers and optimized dilution of CDNA template. PCR amplification was performed with StepOne thermal cycler (ABI, USA). The program included an initial step at $95^{\circ} \mathrm{C}$ for $30 \mathrm{~s}$ followed by 40 cycles of $95^{\circ} \mathrm{C}$ for $15 \mathrm{~s}, 60^{\circ} \mathrm{C}$ for $30 \mathrm{~s}$, and $72^{\circ} \mathrm{C}$ for $10 \mathrm{~s}$. Relative expression of the target genes was calculated by the $2^{-\triangle \Delta C t}$ method. All of the qPCR reactions were performed in triplicate technical repetition, and each with three independent biological repetitions.

\section{Pm60 specific markers and geographic distribution of Pm60 alleles in the WEW population}

Since several conserved SNPs were found that differentiate between functional and nonfunctional TdPm60 alleles, we developed two markers (M-Pm60-S3 and M-Pm60-S4) that can be used for diagnosis of TdPm60 alleles (Fig. S5). KASP marker M-Pm60-S4 can discriminate between the functional (795C), nonfunctional (795T) and null Pm60 alleles. For the M-Pm60-S3 marker, the forward primer targeted site (2335-2354) contained one conserved SNP. The published M-Pm60-S1 marker contains four conserved SNPs, of which three are located on the forward primer (2896-2916) and one on the reverse primer (3944-3966). Therefore, these markers can be used for diagnosing the TdPm60 locus in WEW (see Fig. S5).

A collection of 230 WEW accessions originating from the Fertile Crescent were evaluated for their reaction to Bgt \#15 and were genotyped by three TdPm60 gene-specific markers (M-Pm60-S1, M-Pm60-S3 and MPm60-S4). Geographic distribution of the different alleles was plotted using ggmap R package (Kahle and Wickham, 2013).

\section{Development of introgression lines by marker-assisted selection (MAS)}

PmG16 resistance gene was introgressed from WEW G18-16 to hexaploid wheat after its first transfer to durum wheat var. LDN, following the "durum as a bridge approach" described by Klymiuk et al. (2019). The resistant RIL154 ( $F_{7}$ of the cross of WEW G18-16 with LDN) was selected as a PmG16-donor parent for marker-assisted breeding backcross program which included four backcrosses with bread wheat cultivar Ruta, and one selfing generation to obtain $\mathrm{BC}_{4} \mathrm{~F}_{2}$ introgression lines (ILs). Successful ILs were verified for the presence of the gene using M-Pm60-S1 and uhw390 markers in each generation and further phenotypic validation.

\section{Results}

\section{PmG16 resistance interaction with Bgt isolate \#15}

In a previous study, we have shown that WEW acc. G18-16 was resistant to 34 out of 47 tested $B g t$ isolates from Israel and Switzerland (Ben-David et al. 2010). In the current study we have selected Bgt\#15 to work with since it showed virulence on numerous Pm resistance genes ( $P m 1 a, P m 1 e, P m 2, P m 3 a$, 
Pm3c, Pm3f, Pm3g, Pm4b, Pm5a, Pm5b, Pm6 and Pm7; Ben-David et al. 2010). Plant responses to Bgt \#15 clearly differentiated between the parental lines used in the current study, the WEW acc. G18-16 (highly resistant) and durum cv. LDN (highly susceptible). Small Bgt colonies were observed at 4 days post-infection (dpi) in LDN that rapidly developed massive powdery mildew colonies at 7-10 dpi with infection type (IT) scale 4 (Fig. 1a), as compared to G18-16 that was highly resistant to Bgt \#15 (IT = 0), showing no visible disease symptoms nor signs even at $10 \mathrm{dpi}$ (Fig. 1a). In this incompatible interaction presented by G18-16, histopathology observations have shown that a large number of cells showed $\mathrm{H}_{2} \mathrm{O}_{2}$ accumulation (reddish-brown coloration) and cell death response (blue coloration) already at $2 \mathrm{dpi}$ (10$30 \%$ of the infected epidermis cells in each leaf). In contrast during the compatible interaction, presented by LDN, only $1-3 \%$ of the epidermis cells infected with $\mathrm{Bgt}$ conidia showed slight visible $\mathrm{H}_{2} \mathrm{O}_{2}$ accumulation and cell death response in each leaf at 2 dpi (Fig. 1b and 1c).

Development of fungal structures on wheat leaves of G18-16 and LDN was observed by wheat germ agglutinin conjugated with a fluorophore at a series of time points from 4 to 240 hours post-infection (hpi). The primary germ tubes (PGT), appressorium (App) and haustorial primordia (HP) could be observed in both G18-16 and LDN at 4-, 12- and 24 hpi, respectively (Fig. 1d). However, mature haustorium (MP) and hyphae (Hy) development were visible at 48 hpi only in LDN. Furthermore, the development of conidiophores and massive production of Bgt conidia were observed only in LDN from 240 hpi. In contrast, in G18-16 mature haustoria were not observed and the infection process was halted at less than $48 \mathrm{hpi}$. In only a very few cases (less than 1\%), haustorial primordia were able to develop into mature haustorium and elongated Hy were observed at $72 \mathrm{hpi}$, however, their growth was suppressed 72 to $240 \mathrm{hpi}$ (Fig. 1d). Taken together, these results indicate that PmG16 confers a post-haustoria resistance mechanism against $B g t \# 15$, responsible for restraining the development of mature haustoria and further hyphal growth, accompanied by ROS accumulation and cell death, and resulting in a high resistance response against Bgt \#15.

\section{Genetic mapping of powdery mildew resistance gene PmG16}

A high-density SNP genetic map was recently developed by our lab for the G18-16 × LDN RIL population (147 lines) based on the Illumina Infinium 15K Wheat platform, consisting of 12,905 SNPs (Fatiukha et al. 2019). Using this genetic map, we were able to localize PmG16 on Chr 7AL within a $3.2 \mathrm{cM}$ interval between the proximal flanking marker wsnp_Ku_c28104_38042857 and distal marker RAC875_c8752_1079 (Fig. 2a, Table S1). From a subset of 31 RILs, nine with critical recombination events within the $3.2 \mathrm{cM}$ target interval, were selected and used for further dissection of the target locus using the graphical genotypes approach (Table S2; Young and Tanksley, 1989; Distelfeld et al. 2006). For marker saturation in this region, we used five new SSR markers developed in the current study (Table S3), as well as Xgwm344 and Xwmc809 previously identified as closely linked to PmG16 (Ben-David et al. 2010). Three of the SSR markers (uhw387, uhw388 and uhw389) co-segregated with PmG16 (Fig. 2b, Table S2). Moreover, these markers enabled us to map PmG16 within a $1.4 \mathrm{cM}$, at a distance of 0.3 from marker uhw386 and $1.1 \mathrm{cM}$ from uhw390 (Fig. 2b). 


\section{Comparative genomics analysis of the PmG16 genetic region}

The physical map of PmG16 genetic region was constructed based on the physical positions of the genetic markers anchored to Chr 7AL of WEW genome Zavitan_v.2.0 and T. urartu genome G1812 (Fig. 2c and $2 \mathrm{~d}$ ). The marker positions on the physical map showed complete collinearity with their order on the genetic map, suggesting that the candidate gene region includes approximately $3.48 \mathrm{Mb}$ interval (733339776-736815424 bp in the Chr 7A of Zavitan) flanked by markers uhw386 and uhw390 (Fig. 2c).

To find candidate gene(s) for PmG16, we searched for the annotated genes in the WEW reference genome Zavitan within the $3.48 \mathrm{Mb}$ interval that covers the PmG16 gene region. A total of 34 genes were identified, six of which were annotated as disease resistance (R) proteins with NB-ARC and leucine-rich repeat (LRR) domains, named also as NLRs (nucleotide-binding site leucine-rich repeat) (Table S4). Two candidate NLRs TRIDC7AG076920.1 and TRIDC7AG076930.1 were highly similar to the previously cloned Pm1a (Fig. S1, Table S4). Therefore, we used the functional molecular marker (FMM) Pm1aSTS1 (Hewitt et al. 2020) to genotype G18-16, but it did not yield any amplicons, suggesting that PmG16 is not allelic to Pm1a. Moreover, we found that a third NLR, TRIDC7AG077150.1, gave the highest BLAST scores for the previously cloned $P m 60$ gene located in syntenic location in chromosome $7 \mathrm{~L}$ of $T$. urartu genome (AA), therefore showing close evolutionary genetic relationships (Fig. S1). To support our findings, we genotyped all the RILs that carry critical recombination events and the parental lines with the Pm60 FMM M-Pm60-S1 (Zhao et al. 2020). M-Pm60-S1 showed positive amplification in G18-16 and co-segregated with PmG16 in the RIL population (Fig. S2, Table S2). An additional marker M-Pm60-S3 that was designed to target the Pm60 LRR domain, also showed co-segregation with PmG16 (Fig. S2). Anchoring the genetic markers used in the present study to $T$. urartu chromosome 7AL showed full collinearity with the physical positions of the markers used for the cloning of Pm60 (Zou et al. 2018) (Fig. 2c and 2d). The content and order of all 34 PmG16 candidate genes was highly conserved between the reference genomes of $T$. durum Svevo, and T. dicoccoides Zavitan_V1 and_V2, with only minor rearrangements when compared with $T$. urartu G1812 reference genome (Fig. S3). Therefore, we hypothesized that PmG16 is probably an orthologue of Pm60 in WEW.

\section{Homologous cloning of the TdPm60 locus from WEW}

For further characterization of the Pm60 locus in WEW, we used the homologous cloning strategy for cloning the full length (4365 bp) of the corresponding Pm60 locus (designated as TdPm60 hereafter) from G18-16 genome using several Pm60 cloning primers (Fig. 3a and b). Sequence analysis of TdPm60 identified only eight SNPs in comparison with the original TuPm60 (MF996807.1), and the same length (4365 bp) of mRNA sequence was amplified also from the CDNA of G18-16, suggesting that there are no introns in the gene (Fig. 3c). Six amino acids differed between the putative proteins TdPm60 and TuPm60 (Fig. 3c). Interestingly, no PCR amplification was obtained in LDN using those primers indicating the absence of Pm60 orthologue or high polymorphism in positions of primers between G18-16 and LDN (Fig. 3b). 
Expression patterns of TdPm60 in G18-16 was followed at 12, 24, 36, 48 and 72 hpi with Bgt \#15. The obtained results showed that the relative gene expression of TdPm60 was slightly but significantly ( $p \leq$ 0.05) reduced at 24,48 , and $72 \mathrm{hpi}$ in the Bgt-infected leaves compared with the control set (mockinfected leaves) (Fig. 3d).

\section{Association of Pm60 with Bgt resistance in WEW natural populations and identification of allele} TdPm60a

For testing whether the Pm60 locus is associated with Bgt resistance in WEW natural populations, we used FMM M-Pm60-S1 (Zhao et al. 2020) and additional marker M-Pm60-S3 to screen 230 WEW accessions that originated from across the Fertile Crescent (Fig. 4a and Table 5). Fifty-nine accessions were found to be positive to Pm60 markers and all of them also showed high resistance to Bgt \#15, while all the 67 susceptible accessions showed negative amplification of Pm60 markers (Table 1, Table S5). One hundred and six accessions showed high resistance to Bgt \#15, but were found to be negative to Pm60 markers, indicating the occurrence of other powdery mildew resistance genes in these WEW populations. Previously, the M-Pm60-S1 marker was used for diagnosis of functional Pm60 alleles in the T. urartu natural population and was associated with the Bgt E09 resistance (Zhao et al. 2020). Similarly, our result suggest that the $M-P m 60-S 1$ marker is also associated with Bgt \#15 resistance in the WEW natural population. Moreover, the M-Pm60-S3 could also be used as FMM in the WEW natural population with the same amplification results as M-Pm60-S1.

One resistant accession TD104552 (IT = 1) had a shorter PCR product (Fig. 4a). Sequence analysis revealed a new allele containing a 240-nt deletion (from 3607 to 3368) and two SNPs compared with TdPm60 (T2394G and A3993G), resulting in two amino acid changes (H978Q and T1332A) in the LRR region. Interestingly, this WEW allele, showed the same deletion element previously reported for Pm60a from T. urartu, and therefore it was named TdPm60a.

\section{MIIW72 and MIIW172: potential alleles of TdPm60}

Previous report showed that MIIW72 (Ji et al. 2008), MIWE18 (Han et al. 2009), MIIW172 (Ouyang et al. 2014) and HSM1 (Li et al. 2014) from different Israeli WEW accessions were mapped in the same chromosome region as PmG16 (Ben-David et al. 2010; the terminal bin 0.86-1.00 of Chr 7AL). We anchored the flanking markers of these five genes to the reference genome of WEW. An overlapped chromosome interval was identified on Chr 7AL for these five gene regions (from 735356949 to 736815424) (Fig. 4b). In the overlapped interval, three candidate $R$ genes were identified, TRIDC7AG076920.1 and TRIDC7AG076930.1 which were similar to Pm1a, and TRIDC7AG077150.1 which was similar to Pm60 in the phylogenetic tree (Table S4, Fig. S1). We used the WEW genotypes that were originally used for the identification of the four resistance genes (PmG16, MIIW172, MIIW72 and HSM1) to analyze their Pm1a and Pm60 locus and their response to different Bgt isolates. We found that WEW TD116494 (MIIW172) and TD011558 (MIIW72) accessions were highly resistant to Bgt \#15 with similar resistance spectrum as G18-16, and showed positive amplification for the FMMs M-Pm60-S1 and MPm60-S3 (Fig. 4a, Table S6), while no amplification was obtained for FMM Pm1aSTS1. Sequence 
analysis revealed that TdPm60 in accession MIIW72 had five SNPs (G1242A, A1410C, T2934G, T3017C and A4304G) compared with G18-16, resulting in three amino acid changes (H978Q, P1006L and E1435G). In contrast, accession MIIW172 contained only one SNP (A3525G) as compared with TdPm60 from G18-16, with no amino acid change at the protein level. Those SNPs in the different WEW accessions, which were collected from different places in Israel, probably evolved during host-pathogen co-evolution. TA1410 (HSM1) was partially resistant to Bgt\#15 and Bgt\#101 (IT = 2), but it showed negative PCR amplification for both M-Pm60-S1 and M-Pm60-S3 (Fig. 4a, Table S6). These results indicate that of the five powdery mildew resistance genes, which were mapped in the terminal bin 0.861.00 of 7AL from WEW, at least three of them (PmG16, MIIW72 and MIIW172) may represent different alleles of TdPm60, while MIWE18 locus need to be further analyzed in the future.

\section{Phylogenetic analysis of Pm60 homologs and development of functional KASP marker}

BLAST search of Pm60 homologs in the annotated genome assemblies of domesticated wheat species (T. aestivum, T. spelta, T. durum and T. monococcum) and their closest wild relatives ( $T$. dicoccoides, $T$. urartu and $A$. tauschii), using the GrainGenes and Ensembl Plants website, revealed complex evolutionary relationships at this locus. No similar genes were identified in the genomes of $T$. aestivum cv. Cadenza, Norin 61, CDC Landmark, T. monococcum cv. DV92 and A. tauschii acc. AL8/78. Two genes identified in T. aestivum cv. Chinese Spring (CS), TraesCS7B01G479200 and TraesCS7D01G542200, located on Chr 7B and 7D in the same physical location as Pm60, showed high nucleotide similarity $(96.65 \%$ and $95.59 \%$ Identity, respectively, Table S7) to TdPm60a. In the same location, a truncated gene (1059 bp) with highconfidence annotation was found on chromosome 7A of CS with $77.68 \%$ similarity to Pm60a (Table S7, not included in the phylogenetic tree). We did not find any complete TraesCS7B01G479200 alleles in the 7B chromosomes or any complete Pm60-orthologs in 7A chromosomes from other hexaploid wheat assemblies. In contrast, the TraesCS7D01G542200 alleles from 7D were found in 11 other hexaploid $T$. aestivum and one T. spelta wheat accessions with 0-2 SNPs compared with the gene in CS (Fig. S4). Interestingly, a sequence highly similar to TraesCS7B01G479200 was found in scaffold scfld078262, of $T$. durum cv. Kronos (possibly on 7B) that showed just one SNP (T2281G) and with a missing sequence part (3370-3420 bp). Some other annotated genes (see Fig. 4c) were also identified as Pm60 similar genes (with $73 \sim 80 \%$ identity, 3099-4901 bp) possibly on the same genetic location from different assemblies of related species and were further used for phylogenetic analysis (Table S7). No paralogs of Pm60 was identified from those accessions.

Phylogenetic analysis showed a clear clustering of functional Pm60 alleles and their TdPm60 orthologs (Pm60, Pm60a, Pm60b, TdPm60 and TdPm60a) at both levels of ploidy (AA and AABB genomes) together with the nonfunctional Pm60a'allele from T. urartu acc. PI662227 (Zhao et al. 2018). The two homoeologous genes from T. aestivum cv. CS 7B and 7D genomes were clustered very close to the Pm60 and TdPm60 alleles (Fig. 4c). Moreover, two similar genes from T. urartu acc. G1812 (TRIUR3_00771 and TRIUR3_00770, one gene from T. dicoccoides acc. Zavitan TRIDC7AG077150.1 and an additional identified gene Horvu_FT11_7H01G703800 from barley were grouped on a cluster with longer branches than the Pm60 alleles (Fig. 4c). Interestingly, during the map-based cloning of Pm60, the gene sequences 
TRIUR3_00771 and TRIUR3_00770 (Zou et al. 2018), and TRIDC7AG077150.1 (current study) were identified as the candidate genes of Pm60/TdPm60 from the two susceptible wheat assemblies G1812 and Zavitan (Zou et al. 2018, Table S6). Two genes (TRITD7Av1G277190.1 and TRITD7BV1G230590.1) from the 7A and 7B of T. durum acc. Svevo and two genes (BRADI_1g29658v3 and BRADI_2g38192v3) from Brachypodium distachyon were grouped on two clusters, respectively, and located as an outgroup in the phylogenetic tree (Fig. 4c).

A comparison of protein structures of the functional Pm60 alleles with TraesCS7B01G479200.1, TraesCS7D01G542200.1 and TRIDC7AG077150.1 predicted proteins showed that all of them contained a complete NLR structure. Except TRIDC7AG077150.1, all the Pm60 alleles/homologs had the same motif content and order in the CC and NB-ARC domains, but differ in the motif order and number of motif repeats in the LRR domain (Table S8). Therefore, we can't rule out the option that the differences in motif order and repeat number in the LRR domain may alter the specificity of the NLR to recognize the $B g t$ effector. Nevertheless, CS and Zavitan were susceptible to six Bgt isolates (Table S6), suggesting that TraesCS7B01G479200.1, TraesCS7D01G542200.1 and TRIDC7AG077150.1 are not conferring resistance to those $B g t$ isolates. Moreover, Kronos that harbors a sequence that is highly similar to TraesCS7B01G479200.1 is also susceptible to these six Bgt isolates (Table S6). In contrast, all the functional Pm60 alleles showed highly conserved protein structure (Fig. 4c).

We aligned those identified Pm60 alleles/homologs, based on the SNP at position $795(\mathrm{C} / \mathrm{T})$, designed a KASP marker designated M-Pm60-S4, which enabled us to differentiate between the functional, nonfunctional (possibly located on genomes B and D) and null Pm60 alleles/homologs in the different wheat accessions (Fig. 4d, Fig. S5). For example, 13 WEW accessions containing TdPm60, which were confirmed by marker M-Pm60-S1, showed the functional allele (red circle). Durum cv. LDN and Svevo, bread wheat cv. Fielder and cv. Ruta, and 20 WEW accessions without TdPm60, confirmed by marker $M$ Pm60-S1, showed null M-Pm60-S4 allele, the same as the negative water control (Fig. 4d, Table S5 and S6). CS, Kronos and 6 WEW accessions showed nonfunctional alleles (blue circle), suggesting that those accessions might contain nonfunctional Pm60 alleles/homologs.

\section{Geographic distribution of TdPm60 alleles in WEW populations in the Fertile Crescent}

The markers M-Pm60-S1, M-Pm60-S3 and M-Pm60-S4 were used to screen 230 WEW accessions originating from the Fertile Crescent, including Israel (163), Turkey (28), Syria (16), Iraq (9), Jordan (10) and Lebanon (4), and the resistance of these accessions to Bgt\#15 was checked as well (Table S4). Geographic distribution analysis showed that $T d P m 60$ was only identified in natural populations of WEW from the Southern Levant (52/163 Israel, 3/10 Jordan, 1/4 Lebanon and 2/16 Syria) (Table S5). Of the Israeli accessions, 85.3\% (139/163) were resistant to Bgt, of which $37.4 \%(52 / 139)$ harbor the TdPm60 allele from across all the distribution range of WEW in Israel (Fig. 5). However, most of the WEW accessions from Turkey (Northern Levant) were susceptible with no amplification of the functional Pm60 allele. 
Only one accession in northern Israel contained the TdPm60a allele (with $240 \mathrm{bp}$ deletion) which is regarded as a rare allele, while the $P m 60 \mathrm{~b}$-similar allele (with $240 \mathrm{bp}$ insertion) was not identified among the tested WEW accessions. We have selected 10 accessions containing TdPm60 and one with TdPm60a for the resistance tests to six different $B g t$ isolates. The results showed that all the 11 WEW accessions showed the same resistance spectrum of high resistance to Bgt \#103,\#107, \#101, \#15 and \#70, but susceptible to Bgt \#SH (Table S6). By using KASP marker M-Pm60-S4, 7 Israeli accessions, 1 Syrian and 3 Turkish WEW accessions were identified with nonfunctional alleles/homologs. Among them, 5 accessions were highly susceptible to Bgt \#15 and two of these were further found as susceptible to all the six tested $B g t$ isolates (Table S5 and S6). These results show that nonfunctional alleles/homologs were susceptible to Bgt \#15 or all the six Bgt isolates.

A total of 160 WEW accessions (69.6\%) did not show any amplification by any of the three markers, of which 99 accessions showed resistance to Bgt \#15 suggesting that they probably contain other resistance genes.

\section{Introgression of PmG16 into hexaploid wheat}

As part of the aim to develop resistant pre-breeding genetic resource, we transferred PmG16 into bread wheat cultivar var. Ruta following the "durum as a bridge" approach (Klymiuk et al. 2019). The first step was to test and map the gene in tetraploid durum background and subsequently to introgress the gene by a backcross program into hexaploid cultivar using marker-assisted selection (MAS) (Fig. 6a). The resistant RIL154 ( $F_{7}$ RIL derived from the cross of LDN $\times$ G18-16), was crossed and backcrossed with Ruta to develop $\mathrm{BC}_{4} \mathrm{~F}_{2}$ population accompanied by MAS using the FMM M-Pm60-S1 and the closely linked co-dominant marker uhw390 (Fig. 6b). We tested the introgression lines (ILs) with Bgt \#15, and confirmed that all the ILs (e.g., BC11-15-2, BC12-15-4, BC12-9-2 and BC12-9-3) that showed positive amplification of M-Pm60-S1 were resistant, while all lines with no amplification of this marker (BC12-2-2 and BC12-2-4) were susceptible (Fig. 6b). In addition, since the co-dominant marker uhw390 showed polymorphisms among G18-16, LDN and Ruta, it was used for selecting homozygote introgressions (BC11-15-2, BC12-15-4) resistant to Bgt. The heterozygote lines (BC12-9-2 and BC12-9-3) showed high resistance to $B g t \# 15$, that further confirmed that $P m G 16$ is a dominant gene even in the hexaploid genetic background. The cultivar Ruta was susceptible to four Bgt isolates ( $B g t \# 103, \# 101, \# 70$ and \#15), while resistant to two Bgt isolates \#107 and \#SH, however, the Ruta ILs with PmG16 were highly resistant to all the six tested $B g t$ isolates (Table S6). DAB and Trypan blue staining showed that PmG16 induced ROS accumulation and cell death in the resistant IL BC12-15-4 and halted the development of mature haustorium of $B g t \# 15$; a likely indicator of the activation of a post-haustorium resistance mechanism also in hexaploid wheat. In contrast, in Ruta, mature haustoria and mycelium structures were detected as expected for normal development of Bgt in compatible host-pathogen interactions (Fig. 6c). These results indicate that the dominant gene PmG16 found in WEW is a promising source for bread wheat resistance breeding, and that two markers (M-Pm60-S1 and uhw390) can be used for MAS in the future wheat breeding programs. 


\section{Discussion}

The powdery mildew race-specific resistance gene $P m G 16$, was previously mapped on Chr 7AL of WEW accession G18-16 between the flanking markers Xgwm344 and Xwmc809 (Ben-David et al. 2010). In the current study, based on a high-density SNP map and 8 SSR markers, PmG16 was mapped at a higher precision than in the previous study, which enabled us to narrow down genetic interval that harbors PmG16 to only $1.4 \mathrm{cM}$, residing between flanking markers uhw386 and uhw390 (Fig. 2b). Based on gene annotation of WEW Zavitan genome assembly, six NLR genes have been identified within this interval (Table S4), one of which (TRIDC7AG077150.1) was found at the same syntenic location on chromosome 7A as Pm60, previously cloned in T. urartu (Fig. 2c, 2d and Fig. S3) (Zou et al. 2018). Moreover, we showed that the FMM of Pm60 (M-Pm60-S1) had positive amplification in G18-16 and co-segregated in the mapping population with the resistance conferred by PmG16 (Fig. 4S). Sequence analysis of the cloned gene from G18-16 revealed that it includes eight SNPs as compared with the original Pm60 (MF996807.1), with six amino acids changes at the protein level (Fig. 3c). Hence, we regard the NLR from WEW accession G18-16 as an ortholog of Pm60 from T. urartu and designated it as TdPm60. Screening of 230 WEW from the Fertile Crescent revealed that all the 59 WEW accessions that were positive to amplifications with the Pm60 FMMs (M-Pm60-S1 and M-Pm60-S3) showed high resistance to Bgt\#15, while no susceptible accession had positive amplification of the FMMs (Table 1). Since the linkage disequilibrium (LD) in WEW decays very rapidly (Sela et al. 2014, Sela et al. unpublished), it is unlikely that other genes in the interval are PmG16. Altogether, the physical location of the gene, sequence alignments and specific Pm60 marker analyses suggest that TdPm60 is an ortholog of Pm60, a candidate gene for PmG16, associated with Bgt resistance in WEW natural populations. Ultimately, a conclusive evidence that the encoding TdPm60 sequence confers PmG16 resistance needs to be substantiated by future work using loss-of function mutants or transgenic complementation.

In the current study, we have shown that $T d P m 60$ (PmG16) confers a post-haustorium resistance mechanism against Bgt\#15, blocking the development of mature haustorium and hyphal growth. TdPm60 induced ROS accumulation and cell death defense responses, both in tetraploid and hexaploid backgrounds, similar to those induced by Pm60 in the infected T. urartu leaves (Zou et al. 2018; Zhao et al. 2020), resulting in high resistance to Bgt \#15 (Fig. 1a-d). The same post-haustorium resistance mechanism has also been found in WEW accession G305-3M which contains PmG3M (Wei et al. 2020). Most $P m$ genes cloned so far are induced by $B g t$ at different time points from 6 to $48 \mathrm{hpi}$, such as $P m 5 e$ (Xie et al. 2020), Pm41(Li et al. 2020), Pm21(He et al. 2018), Pm2 (Chen et al. 2019), and Pm24 (Lu et al. 2020). On the contrary, the expression of WTK1 ( Yr15) also derived from WEW is down-regulated by the presence of the yellow rust pathogen (Klymiuk et al. 2018). The expression of Pm60 in T. urartu does not significantly change by Bgt infection at 1-5 dpi (Zou et al. 2018), while the expression level of TdPm60 was slightly but significantly ( $\mathrm{x} \leq 0.05$ ) reduced at 24,48 , and 72 hpi in Bgt-infected G18-16 leaves compared with mock-inoculated leaves (Fig. 3d).

A few cloned resistance genes in wheat were found to be orthologous to already cloned resistance genes even at different ploidy levels. For example, Pm3b cloned from Chr $1 \mathrm{~A}$ of hexaploid wheat, which encode 
a CC-NBS-LRR and confer race-specific resistance to powdery mildew (Yahiaoui et al. 2004), was found to be orthologous to other genes based on homology cloning or physical and genetic mapping. The resistance allele $P m 3 \mathrm{k}$ from tetraploid wheat, and $P m 8$ from rye have been characterized as the orthologues of the Pm3 allelic series (Yahiaoui et al. 2009; Hurni et al. 2013). Moreover, several genes that were previously mapped to the same interval in different mapping populations were found to belong to a single locus. For example, Klymiuk et al. (2018) cloned the stripe rust resistance gene Yr15 from WEW G25, and later found that the genes $\mathrm{YrH} 52$ and $\mathrm{YrG303}$, originated from different accessions, represent the same gene (Klymiuk et al. 2020). More than 10 powdery mildew resistance genes have been previously identified in the Xwmc525-Xgwm344 genetic interval on Chr 7A, including Pm1a, Pm1c and Pm1e (syn. Pm22) from bread wheat (Hsam et al. 1998; Neu et al. 2002), Pm1d from T. spelta (Hsam et al. 1998), Pm1b, MIm2033 and MIm80 from T. monococcum (Yao et al. 2007), PmTb7A.2 from T. boeoticum (Chhuneja et al. 2015), Pm60 (PmU) from T. urartu (Zou et al. 2018; Zhang et al. 2018), and MIAG12 from T. timopheevii (Maxwell et al. 2009). Five genes from WEW were also identified in this region, including MIIW72 (Ji et al. 2008), HSM1 (Li et al. 2014), MIIW172 (Ouyang et al. 2014), MIWE18 (Han et al. 2009) and PmG16 (Ben-David et al. 2010; and the current study). For the five genes identified in WEW, further sequencing analysis revealed that at least three of them (MIIW172, MIIW72 and PmG16 in current study) might be orthologs of Pm60 from T. urartu (Fig. 4b). Pm1a and Pm60 have been demonstrated as different genes in the same genetic region (Hewitt et al. 2020). Using Pm1a BLAST search in GrainGenes database, we identified six Pm1a homologs (Identity $=77.7-88.5 \%$ ) in six different reference wheat genomes, which were present at 0.21-1.87 Mb apart from the Pm60 homologs towards the centromeric positions (Table S9 and S10). Additionally, we excluded the possibility of PmG16, MIIW72, MIIW172 and HSM1 as the functional Pm1a alleles by using FMM Pm1aSTS1. Further studies are needed to determine whether the other $P m$ genes which mapped to same genetic region, such as $P m 1 b$-e, are truly allelic to Pm1a or possibly the orthologs of $P m 60$, or may even represent novel $P m$ genes.

Pm60 was cloned from T. urartu by positional cloning, while Pm60b and Pm60a were identified in $T$. urartu natural populations by marker selection and homology cloning (Zou et al. 2018). The accessions containing Pm60, Pm60b or Pm60a showed high resistance to BgtE09, and all the three alleles induced cell death in N. benthamiana (Zou et al. 2018; Zhao et al. 2020). Genetic mapping in $F_{2}$ segregating populations suggested that $P m 60 b$ is a functional allele with 240 bp nucleotide (nt) insertion in the LRR region (Zhao et al. 2020). In the current study, 58 WEW accessions had the TdPm60 allele and only one (TD1004552) had the TdPm60a allele, while TdPm60b was not found in the WEW natural populations. The nonfunctional allele Pm60a'that had 58 SNPs and a 3-nt deletion was identified in 9 susceptible $T$. urartu accessions, suggesting that these SNPs and 3-nt deletion may be important for the resistance function (Zhao et al. 2020). In our study, we identified a resistant WEW accession (TD1004552) that had a TdPm60a allele with a 240-nt deletion and one SNP in the LRR region compared with TdPm60 (Fig. 4a). Interestingly, compared with Pm60a from T. urartu, TdPm60a has the same deletion as Pm60a but almost the same SNPs as TdPm60 suggesting convergent molecular evolution of $T d P m 60$ alleles in WEW. Therefore, it seems that the same deletion of 240-nt occurred twice, maybe due to the presence of 
repetitive sequences in this genomic region. The repetitive sequence may have caused additional indel in the Pm60b allele. This LRR length variation caused by repeats is one of the main sources for NLR variability (Wicker et al. 2007). The deletion in WEW accession TD1004552 (TdPm60a) showed similar resistance spectrum as G18-16, carrying the TdPm60 allele, however, it is also possible that another Pm gene is present in this accession, and further studies are still needed to verify the TdPm60a function.

Based on BLAST analysis with published sequence data of several wheat cultivars, we found that although the $P m 60$ homologs are highly polymorphic, the functional alleles are highly conserved (Fig. 4c). However, some similar sequences ( $\geq 95.59 \%$ ) of $P m 60$ could be identified on several susceptible wheat accessions (i.e, Kronos and Chinese Spring; Fig. S5). The sequences found in the domesticated cultivars and CS are probably homoeologous sequences located on genomes $B$ and $D$. These sequences have a deletion of $240 \mathrm{nt}$ in the LRR domain relative to Pm60 (the same as the Pm60a allele) with different motif order and different number of repeats (Table S8). However, these sequences differ from Pm60 by only 4\% and maintain the reading frame and the important motives in the NBS domain. Therefore, they may be functional against other Bgt races or other pathogens although the CS was susceptible to all of the six tested $B g t$ \# isolates. Sequence diversity in the LRR region was previously demonstrated for different functional Pm3 alleles (Bhullar et al. 2009). These findings support the suggested role of the LRR domain as an effector-binding and regulatory domain in recognition specificity during the coevolution of pathogen effectors and host NLR receptors (DeYoung et al. 2006).

Previous report showed that $T$. urartu accessions carrying the Pm60 allele are present mostly in Lebanon (28/83) and Syria (14/39) (Zhao et al. 2020), while T. urartu accessions collected in Turkey were more susceptible to $B g t$ and contained very few Pm60 alleles (3/77). In the current study, we did not identify any functional Pm60 allele in 29 Turkish WEW accessions. Among them, just two accessions were resistant to Bgt \#15 (Fig. 5 and Table S5). In contrast, $30 \%$ of the WEW accessions collected in the Southern Levant contained TdPm60 (Fig. 5 and Table S4). Therefore, in both T. urartu and WEW natural populations, the resistant accessions baring Pm60 are highly abundant in the Southern Levant as compared with the Northern Levant regions. Previous reports using SNP based clustering show that WEW accessions from Southern (Israel, Syria, and Lebanon) and Northern (Turkey) Levant are clustered separately (Avni et al. 2017), and the same pattern is observed in T. urartu (Ling et al. 2018), which is the diploid $(2 n=A A)$ progenitor of the A sub-genome of WEW. Therefore, $P m 60$ in WEW was possibly inherited from $T$. urartu by the polyploidization event that gave birth to WEW and took place in the Southern Levant (Feldman and Kislev, 2007). Interestingly, a previous study in T. monococcum $(2 \mathrm{n}=\mathrm{AA})$ natural populations ( 270 accessions) showed that six accessions carried the Pm60 allele (Shen et al. 2018). Supported by the previous results, the current study suggest that $P m 60$ is an ancient gene present in three Triticum species where it shows a high level of presence/absence polymorphism typical of some R genes (Shen et al. 2006). It is possible that the low frequency of Pm60 in the Northern Levant is caused by natural selection because it does not have a selective advantage there. More evidence is needed to elucidate the genetic evolution of $\mathrm{Pm60}$ in the A genome species. The distribution and variation of the functional Pm60 alleles in the domesticated emmer, durum, and common wheat should be further studied using the newly developed KASP markers, as well as the other three functional SSR markers ( $M$ - 
Pm60-S1, M-Pm60-S2 and M-Pm60-S3). We believe that the Pm60, as an ancient and transferable NLR gene, can be used as a classical locus for understanding the evolution and variation of the NLR gene families in wheat. These findings can be explained also by frequent alien introgression to domesticated wheat from some relative species (Zhou et al. 2020).

Transferring of Pm60 from T. urartu into susceptible bread wheat cv. Kn199 by genetic transformation conferred high resistance to Bgt (Zou et al. 2018). In the current study, we successfully developed several ILs which carry PmG16 and showing high resistance to Bgt\#15, suggesting that PmG16 from WEW could confer resistance in both tetraploid and hexaploid genetic backgrounds and there is no suppression of the PmG16 resistance by the D genome. PmG16 induced ROS and cell death defense responses in both G18-16 and the hexaploid ILs, suggesting that PmG16 resistance response is expressed as a typical NLR gene both in WEW and in bread wheat backgrounds (Fig. 1b, c and Fig. 6c). Therefore, Pm60 orthologs are promising genetic sources for bread wheat resistance breeding. One dominant marker $M$ Pm60-S1 could be used for MAS in those introgression lines, while the co-dominant SSR marker uhw390 which is linked to PmG16 could be used for selection of homozygotes during MAS (Fig. 6). Moreover, since WEW is an excellent source for other disease resistance genes/alleles, in future breeding program we propose to pyramid several such genes from WEW and transfer them into durum and bread wheat varieties to obtain more durable resistance to different diseases. These genes can include powdery mildew resistance genes PmG16 and PmG3M (Xie et al. 2012; Wei et al. 2020), and the broad spectrum yellow rust resistance genes from WEW, such as Yr15 (Klymiuk et al. 2018) and Yr36 (Fu et al. 2009). Altogether, the results of our study demonstrate that WEW is a valuable genetic resource for improving disease resistance in wheat cultivars.

\section{Declarations}

Author Contributions: Conceived and designed the study, T.F., Y.L., Z.W.; Development of the critical RILs, Z.W., A.F. and Y.L.; Data analysis of genotyping by $15 \mathrm{~K}$ wheat SNP arrays of Trait Genetics ${ }^{\circledR}$, A.F., Z.W. and Y.L.; Marker development and analysis, H.W., Z.W., S.J. and Y.L.; Microscopic observations, Y.L., Z.W. and S.J.; Phenotyping, Z.W., Y.L., S.H. and S.J.; Sequencing, Y.L. and S.J.; Germplasm selection, T.K. and Y.L.; Bioinformatic analysis of Pm60 locus, A.F., Y.L. and H.S.; Writing manuscript, Y.L. and T.F.; Reviewing and editing, A.F., H.S., Z.W., T.K. and T.F.; coordination and funding acquisition, T.F.

Funding: This study was supported by the Israel Science Foundation, grant number 1719/08 and 1366/18.

Acknowledgements: Y.L. was supported by a fellowship provided by the Planning and Budgeting Committee (PBC) of the Israel Council for Higher Education for Outstanding Post-doctoral Fellows from China and India - from 2018 to 2021. Z.W. was supported by the China Scholarship Council for financial support and the University of Haifa Graduate School. The authors wish to thank Dr. Meng Xu for development of the geographic distribution map, Dr. Tamar Kis-papo for her technical support and Dr. Rajib Roychowdhury for critical reading and English editing of the manuscript. 
Conflicts of Interest: The authors declare no conflict of interest.

\section{References}

Asif M, lqbal M, Randhawa H, Spaner D (2014) Wheat: The miracle cereal. In Managing and Breeding Wheat for Organic Systems, Springer, Cham, Berlin, Germany, pp 1-7

Avni R, Nave M, Barad O, Baruch K, Twardziok SO, Gundlach H, Hale I, Mascher M, Spannagl M, Wiebe K, Jordan KW (2017) Wild emmer genome architecture and diversity elucidate wheat evolution and domestication. Science 357(6346):93-97

Ben-David R, Xie W, Peleg Z, Saranga Y, Dinoor A and Fahima T (2010) Identification and mapping of PmG16, a powdery mildew resistance gene derived from wild emmer wheat. Theoretical and Applied Genetics 121(3): 499-510

Bhullar NK, Street K, Mackay M, Yahiaoui N and Keller B (2009) Unlocking wheat genetic resources for the molecular identification of previously undescribed functional alleles at the Pm3 resistance locus. Proceedings of the National Academy of Sciences 106(23):9519-9524

Camacho C, Coulouris G, Avagyan V, Ma N, Papadopoulos J, Bealer K and Madden TL (2009) BLAST+: architecture and applications. BMC Bioinformatics 10(1):421.

Chhuneja P, Yadav B, Stirnweis D, Hurni S, Kaur S, Elkot AF, Keller B, Wicker T, Sehgal S, Gill BS and Singh K (2015) Fine mapping of powdery mildew resistance genes PmTb7A.1 and PmTb7A. 2 in Triticum boeoticum (Boiss.) using the shotgun sequence assembly of chromosome 7AL. Theoretical and Applied Genetics 128(10):2099-2111

DeYoung BJ and Innes RW (2006) Plant NBS-LRR proteins in pathogen sensing and host defense. Nature Immunology 7(12):1243-1249

Distelfeld A, Uauy C, Fahima T and Dubcovsky J (2006) Physical map of the wheat high-grain protein content gene $G p c-B 1$ and development of a high-throughput molecular marker. New Phytologist 169(4):753-763

Feldman M and Kislev ME (2007) Domestication of emmer wheat and evolution of free-threshing tetraploid wheat. Israel Journal of Plant Sciences 55(3-4):207-221

Fatiukha A, Deblieck M, Klymiuk V, Merchuk-Ovnat L, Peleg Z, Ordon F, Fahima T, Korol AB, Saranga Y and Krugman $T$ (2019) Genomic architecture of phenotypic plasticity of complex traits in tetraploid wheat in response to water stress. bioRxiv, 565820

Han J, Zhang L, Li G, Zhang H, Xie C, Yang Z, Sun Q and Liu Z (2009) Molecular mapping of powdery mildew resistance gene MIWE18 in wheat originated from wild emmer (Triticum turgidum van dicoccoides). Acta Agronomica Sinica 35(10):1791-1797 
He H, Zhu S, Zhao R, Jiang Z, Ji Y, Ji J, Qiu D, Li H and Bie T (2018) Pm21, encoding a typical CC-NBSLRR protein, confers broad-spectrum resistance to wheat powdery mildew disease. Molecular Plant $11(6): 879-882$

Hewitt T, Mueller MC, Molnár I, Mascher M, Holušová K, Šimková H, Kunz L, Zhang J, Li J, Bhatt D, Sharma R, Schudel S, Yu G, Steuernagel B, Periyannan S, Wulff B, Ayliffe M, Mclntosh R, Keller B, Lagudah E and Zhang P (2020) A highly differentiated region of wheat chromosome 7AL encodes a Pm1a immune receptor that recognises its corresponding AvrPm1a effector from Blumeria graminis. New Phytologist, doi: 10.1111/nph.17075

Hsam SLK and Zeller FJ (1997) Evidence of allelism between genes Pm8 and Pm17 and chromosomal location of powdery mildew and leaf rust resistance genes in the common wheat cultivar 'Amigo'. Plant Breeding 116(2):119-122

Hsam SLK, Huang XQ, Ernst F, Hartl L and Zeller FJ (1998) Chromosomal location of genes for resistance to powdery mildew in common wheat (Triticum aestivum L. em Thell.) 5 Alleles at the Pm1 locus. Theoretical and Applied Genetics 96(8):1129-1134

Huang L, Raats D, Sela H, Klymiuk V, Lidzbarsky G, Feng L, Krugman T and Fahima, T (2016) Evolution and adaptation of wild emmer wheat populations to biotic and abiotic stresses. Annual Review of Phytopathology 54:279-301

Hurni S, Brunner S, Buchmann G, Herren G, Jordan T, Krukowski P, Wicker T, Yahiaoui N, Mago R and Keller B (2013) Rye Pm8 and wheat Pm3 are orthologous genes and show evolutionary conservation of resistance function against powdery mildew. The Plant Journal 76(6):957-969

Ji X, Xie C, Ni Z, Yang T, Nevo E, Fahima T, Liu Z and Sun Q (2008) Identification and genetic mapping of a powdery mildew resistance gene in wild emmer (Triticum dicoccoides) accession IW72 from Israel. Euphytica 159(3):385-390

Joehanes R and Nelson JC (2008) QGene 4.0, an extensible Java QTL-analysis platform. Bioinformatics 24(23):2788-2789

Jupe F, Pritchard L, Etherington GJ, MacKenzie K, Cock PJ, Wright F, Sharma SK, Bolser D, Bryan GJ, Jones JD and Hein I (2012) Identification and localisation of the NB-LRR gene family within the potato genome. BMC Genomics 13(1):75

Kahle D and Wickham H (2013) ggmap: Spatial Visualization with ggplot2. The R Journal 5(1):144-161

Klymiuk V, Fatiukha A, Huang L, Wei Z, Kis-Papo T, Saranga Y, Krugman T and Fahima T (2019) Durum wheat as a bridge between wild emmer wheat genetic resources and bread wheat. Applications of Genetic and Genomic Research in Cereals. Woodhead Publishing pp201-230 
Klymiuk V, Fatiukha A, Raats D, Bocharova V, Huang L, Feng L, Jaiwar S, Pozniak C, Coaker G, Dubcovsky $\mathrm{J}$ and Fahima $\mathrm{T}$ (2020) Three previously characterized resistances to yellow rust are encoded by a single locus Wtk1. Journal of Experimental Botany 71(9):2561-2572

Klymiuk V, Yaniv E, Huang L, Raats D, Fatiukha A, Chen S, Feng L, Frenkel Z, Krugman T, Lidzbarsky G and Chang W (2018) Cloning of the wheat Yr15 resistance gene sheds light on the plant tandem kinasepseudokinase family. Nature Communications 9(1):1-12

Krattinger SG, Lagudah ES, Spielmeyer W, Singh RP, Huerta-Espino J, McFadden H, Bossolini E, Selter LL and Keller $B$ (2009) A putative $A B C$ transporter confers durable resistance to multiple fungal pathogens in wheat. Science 323(5919):1360-1363

Kumar S, Stecher G, Li M, Knyaz C and Tamura K (2018) MEGA X: Molecular Evolutionary Genetics Analysis across computing platforms. Molecular Biology and Evolution 35:1547-1549

Li M, Dong L, Li B, Wang Z, Xie J, Qiu D, Li Y, Shi W, Yang L, Wu Q and Chen Y. (2020) A CNL protein in wild emmer wheat confers powdery mildew resistance. New Phytologist 228:1027-1037

Li N, Wen Z, Wang J, Fu B, Liu J, Xu H, Kong Z, Zhang L, Jia H and Ma Z (2014) Transfer and mapping of a gene conferring later-growth-stage powdery mildew resistance in a tetraploid wheat accession. Molecular Breeding 33(3):669-677

Liang J, Fu B, Tang W, Khan NU, Li N and Ma Z (2016) Fine mapping of two wheat powdery mildew resistance genes located at the Pm1 cluster. The Plant Genome 9(2):1-9

Ling H, Ma B, Shi X, Liu H, Dong L, Sun H, Cao Y, Gao Q, Zheng S, Li Y and Yu Y (2018) Genome sequence of the progenitor of wheat A subgenome Triticum urartu. Nature 557(7705):424-428

Lu P, Guo L, Wang Z, Li B, Li J, Li Y, Qiu D, Shi W, Yang L, Wang N and Guo G (2020) A rare gain of function mutation in a wheat tandem kinase confers resistance to powdery mildew. Nature Communications 11(1):1-11

Maccaferri M, Harris NS, Twardziok SO, Pasam RK, Gundlach H, Spannagl M, Ormanbekova D, Lux T, Prade VM, Milner SG and Himmelbach A (2019) Durum wheat genome highlights past domestication signatures and future improvement targets. Nature Genetics 51(5):885-895

Maxwell JJ, Lyerly JH, Cowger C, Marshall D, Brown-Guedira G and Murphy JP (2009) MIAG12: a Triticum timopheevii-derived powdery mildew resistance gene in common wheat on chromosome 7AL. Theoretical and Applied Genetics 119(8):1489-1495

Mclntosh RA, Dubcovsky J, Rogers WJ, et al. (2019) Catalogue of gene symbols for wheat: 2019 Supplement. Annul Wheat Newsletter 65:98-109 
Moore JW, Herrera-Foessel S, Lan C, Schnippenkoetter W, Ayliffe M, Huerta-Espino J, Lillemo M, Viccars L, Milne R, Periyannan S and Kong X (2015) A recently evolved hexose transporter variant confers resistance to multiple pathogens in wheat. Nature Genetics 47(12):1494-1498

Neu C, Stein N and Keller B (2002) Genetic mapping of the Lr20 Pm1 resistance locus reveals suppressed recombination on chromosome arm 7AL in hexaploid wheat. Genome 45(4):737-744

Ouyang S, Zhang D, Han J, Zhao X, Cui Y, Song W, Huo N, Liang Y, Xie J, Wang Z and Wu Q (2014) Fine physical and genetic mapping of powdery mildew resistance gene MIIW172 originating from wild emmer (Triticum dicoccoides). Plos One 9(6):e100160

Peleg Z, Saranga Y, Suprunova T, Ronin Y, Röder MS, Kilian A, Korol AB and Fahima T (2008) High-density genetic map of durum wheat $\times$ wild emmer wheat based on SSR and DArT markers. Theoretical and Applied Genetics 117(1):103

Porebski S, Bailey LG, Baum BR (1997) Modification of a CTAB DNA extraction protocol for plants containing high polysaccharide and polyphenol components. Plant Molecular Biology Reporter 15:8-15

Qiu Y, Zhou R, Kong X, Zhang S and Jia J (2005) Microsatellite mapping of a Triticum urartu Tum. derived powdery mildew resistance gene transferred to common wheat (Triticum aestivum $L$.). Theoretical and Applied Genetics 111(8):1524-1531

Sánchez-Martín J, Steuernagel B, Ghosh S, Herren G, Hurni S, Adamski N, Vrána J, Kubaláková M, Krattinger SG, Wicker T and Doležel J (2016) Rapid gene isolation in barley and wheat by mutant chromosome sequencing. Genome Biology 17(1):221

Sela H, Ezrati S, Ben-Yehuda P, Manisterski J, Akhunov E, Dvorak J, Breiman A and Korol A (2014) Linkage disequilibrium and association analysis of stripe rust resistance in wild emmer wheat (Triticum turgidum ssp. dicoccoides) population in Israel. Theoretical and Applied Genetics 127(11):2453-2463

Shen J, Araki H, Chen L, Chen J and Tian D (2006) Unique evolutionary mechanism in R-genes under the presence/absence polymorphism in Arabidopsis thaliana. Genetics 172(2):1243-1250

Shen Q, Li Y, Zhao F, and Zhou L (2018) Development and application of specific molecular markers for identification of powdery mildew resistance gene Pm60. China patent CN 108950057A

Singh RP, Singh PK, Rutkoski J, Hodson DP, He X, Jørgensen, LN, Hovmøller, MS and Huerta-Espino J (2016) Disease impact on wheat yield potential and prospects of genetic control. Annual Review of Phytopathology 54:303-322

Steuernagel B, Witek K, Krattinger SG, Ramirez-Gonzalez RH, Schoonbeek HJ, Yu G, Baggs E, Witek Al, Yadav I, Krasileva, KV and Jones JD (2020) The NLR-Annotator tool enables annotation of the intracellular immune receptor repertoire. Plant Physiology 183(2):468-482 
Tang D and Zou S (2017) Wheat powdery mildew resistant gene PmR2 and its clone and application. China patent CN201710632539.2A

Thordal-Christensen $\mathrm{H}$, Zhang Z, Wei Y and Collinge DB (1997) Subcellular localization of $\mathrm{H}_{2} \mathrm{O}_{2}$ in plants: $\mathrm{H}_{2} \mathrm{O}_{2}$ accumulation in papillae and hypersensitive response during the barley-powdery mildew interaction. The Plant Journal 11(6):1187-1194

Van Ooijen JW (2006) JoinMap 4. Software for the calculation of genetic linkage maps in experimental populations. Kyazma BV, Wageningen, Netherlands, 33

van Wees S (2008) Phenotypic analysis of Arabidopsis mutants: trypan blue stain for fungi, oomycetes, and dead plant cells. Cold Spring Harbor Protocols 8: pdb-prot4982

Voorrips RE (2002) MapChart: software for the graphical presentation of linkage maps and QTLs. Journal of Heredity 93(1):77-78

Walkowiak S, Gao L, Monat C, Haberer G, Kassa MT, Brinton J, et al. (2020) Multiple wheat genomes reveal global variation in modern breeding. Nature 588:277-283

Wei Z, Klymiuk V, Bocharova V, Pozniak C. and Fahima T (2020) A post-haustorial defense mechanism is mediated by the powdery mildew resistance gene, PmG3M, derived from wild emmer wheat. Pathogens $9(6): 418$

Wicker T, Yahiaoui N and Keller B (2007) Illegitimate recombination is a major evolutionary mechanism for initiating size variation in plant resistance genes. The Plant Journal 51(4):631-641

Xie J, Guo G, Wang Y, Hu T, Wang L, Li J, Qiu D, Li Y, Wu Q, Lu P, Chen Y, et al (2020) A rare single nucleotide variant in Pm5e confers powdery mildew resistance in common wheat. New Phytologist 228:1011-1026

Xie W, Ben-David R, Zeng B, Distelfeld A, Röder MS, Dinoor A and Fahima T (2012) Identification and characterization of a novel powdery mildew resistance gene PmG3M derived from wild emmer wheat, Triticum dicoccoides. Theoretical and Applied Genetics 124(5):911-922

Xing L, Hu P, Liu J, Witek K, Zhou S, Xu J, Zhou W, Gao L, Huang Z, Zhang R and Wang X (2018) Pm21 from Haynaldia villosa encodes a CC-NBS-LRR protein conferring powdery mildew resistance in wheat. Molecular Plant 11(6):874-878

Xu X, Li Q, Ma Z, Fan J and Zhou Y (2018) Molecular mapping of powdery mildew resistance gene $P m S G D$ in Chinese wheat landrace Shangeda using RNA-seq with bulk segregant analysis. Molecular Breeding 38(3):23

Yahiaoui N, Kaur N and Keller B (2009) Independent evolution of functional Pm3 resistance genes in wild tetraploid wheat and domesticated bread wheat. The Plant Journal 57(5):846-856 
Yahiaoui N, Srichumpa P, Dudler R and Keller B (2004) Genome analysis at different ploidy levels allows cloning of the powdery mildew resistance gene $P m 3 b$ from hexaploid wheat. The Plant Journal 37(4):528-538

Yao G, Zhang J, Yang L, Xu H, Jiang Y, Xiong L, Zhang C, Zhang Z, Ma Z and Sorrells ME (2007) Genetic mapping of two powdery mildew resistance genes in einkorn (Triticum monococcum L.) accessions. Theoretical and Applied Genetics 114(2):351-358

Zhang D, Zhu K, Dong L, Liang Y, Li G, Fang T, Guo G, Wu Q, Xie J, Chen Y, Lu P, et al (2019) Wheat powdery mildew resistance gene Pm64 derived from wild emmer (Triticum turgidum var. dicoccoides) is tightly linked in repulsion with stripe rust resistance gene Yr5. The Crop Journal 7(6):761-770

Zhang L, Zheng X, Qiao L, Qiao L, Zhao J Wang J and Zheng J (2018) Analysis of three types of resistance gene analogs in PmU region from Triticum urartu. Journal of Integrative Agriculture 17(12):2601-2611

Zhao F, Li Y, Yang B, Yuan H, Jin C, Zhou L, Pei H, Zhao L, Li Y, Zhou Y, Xie J and Shen Q (2020) Powdery mildew disease resistance and marker-assisted screening at the Pm60 locus in wild diploid wheat Triticum urartu. The Crop Journal 8(2):252-259

Zhou Y, Zhao X, Li Y, Xu J, Bi A, Kang L, Xu D, Chen H, Wang Y, Wang Y, Liu S, Jiao C, Lu H, Wang J, Yin C, Jiao $Y$ and Lu $F(2020)$ Triticum population sequencing provides insights into wheat adaptation. Nature Genetics 52:1412-1422

Zhu T, Wang L, Rodriguez JC, Deal KR, Avni R, Distelfeld A, McGuire PE, Dvorak J and Luo MC (2019) Improved genome sequence of wild emmer wheat Zavitan with the aid of optical maps. G3: Genes, Genomes, Genetics 9(3):619-624

Zou S, Wang H, Li Y, Kong Z and Tang D (2018) The NB-LRR gene Pm60 confers powdery mildew resistance in wheat. New Phytologist 218(1):298-309

\section{Tables}

Table 1 Distribution of Pm60 among resistant (R) and susceptible (S) WEW accessions.

\begin{tabular}{|llll|}
\hline & \multicolumn{3}{c|}{ Pm60 } \\
\hline & & + & - \\
\hline Reaction to & $\mathrm{R}$ & 59 & 106 \\
Bgt \#15 & $\mathrm{S}$ & 0 & 66 \\
\hline
\end{tabular}

Note: " $R$ " indicates resistant; " $S$ " indicates susceptible; "-" indicates negative amplification and "+" indicates positive amplification of M-Pm60-S1 and M-Pm60-S3 markers. 


\section{Figures}
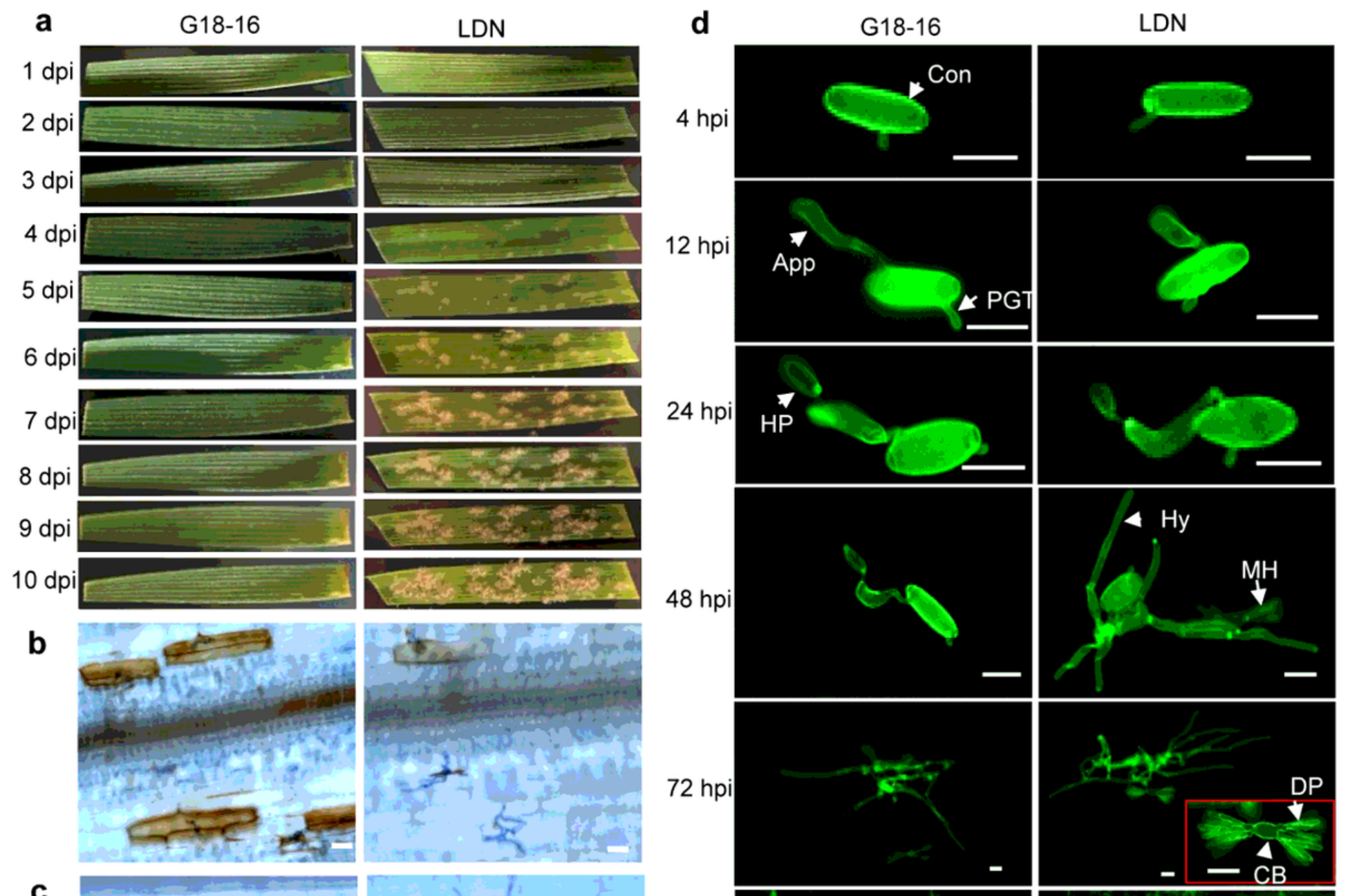

b
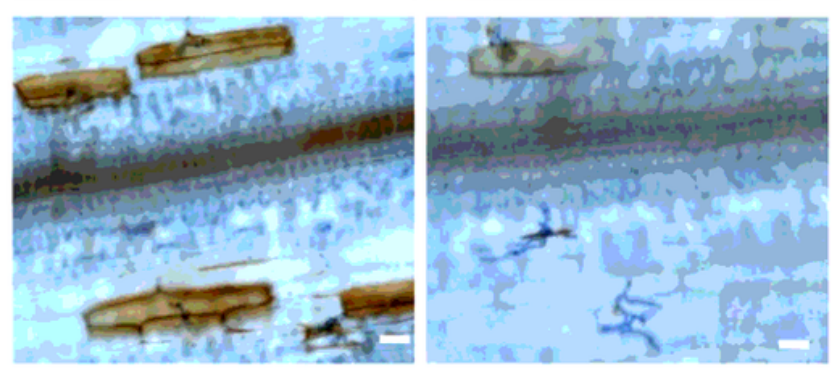

c
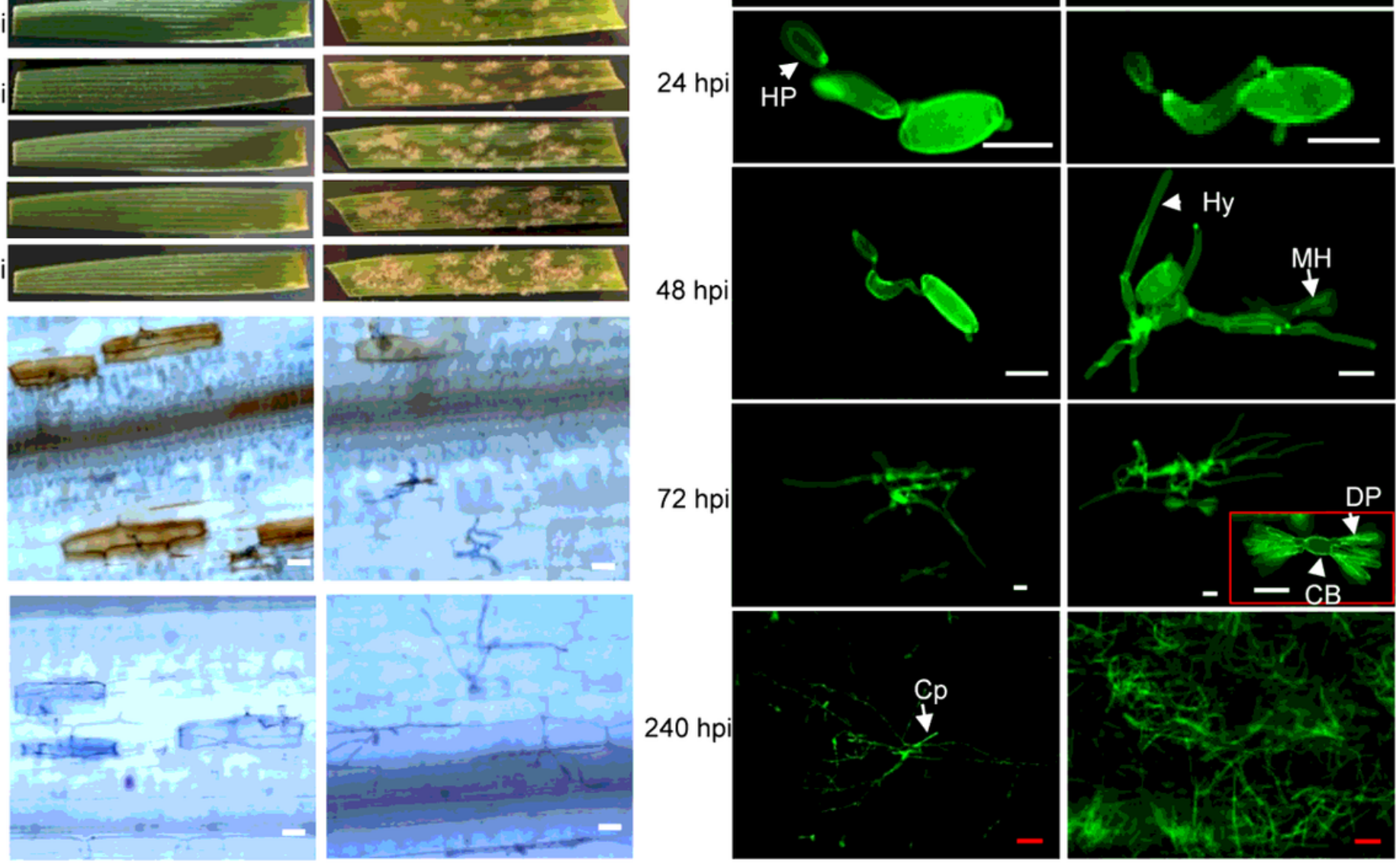

\section{Figure 1}

Bgt \#15 development on the young leaves of WEW G18-16 and durum LDN, ROS accumulation and cell death resistance response. a Macroscopic observation of the response of G18-16 and LDN leaves to Bgt infection 1 to 10 days post-infection (dpi); b DAB staining of leaves infected with Bgt \#15 at 2 dpi. Reddish-brown coloration shows the accumulation of H2O2. Bars $=50 \mu \mathrm{m} ; \mathrm{c}$ Trypan blue staining of leaves used to visualize fungal structures and plant cell death with blue coloration. Bars $=50 \mu \mathrm{m} ; \mathrm{d}$ Microscopic observation of Bgt-wheat interactions in the G18-16 and LDN inoculated leaves. These leaf samples were visualized under a fluorescence microscope after staining with wheat germ agglutinin (WGA) at six time points post-inoculation. App, appressorium; CB, central body; Con, conidium; $\mathrm{Cp}$, 
conidiophore; DP, digitate processes; HP, haustorial primordia; Hy, hyphae; $\mathrm{MH}$, mature haustorium; PGT, primary germ tube. Bars $=20 \mu \mathrm{m}(4-72 \mathrm{hpi})$ and $100 \mu \mathrm{m}(240 \mathrm{hpi})$.

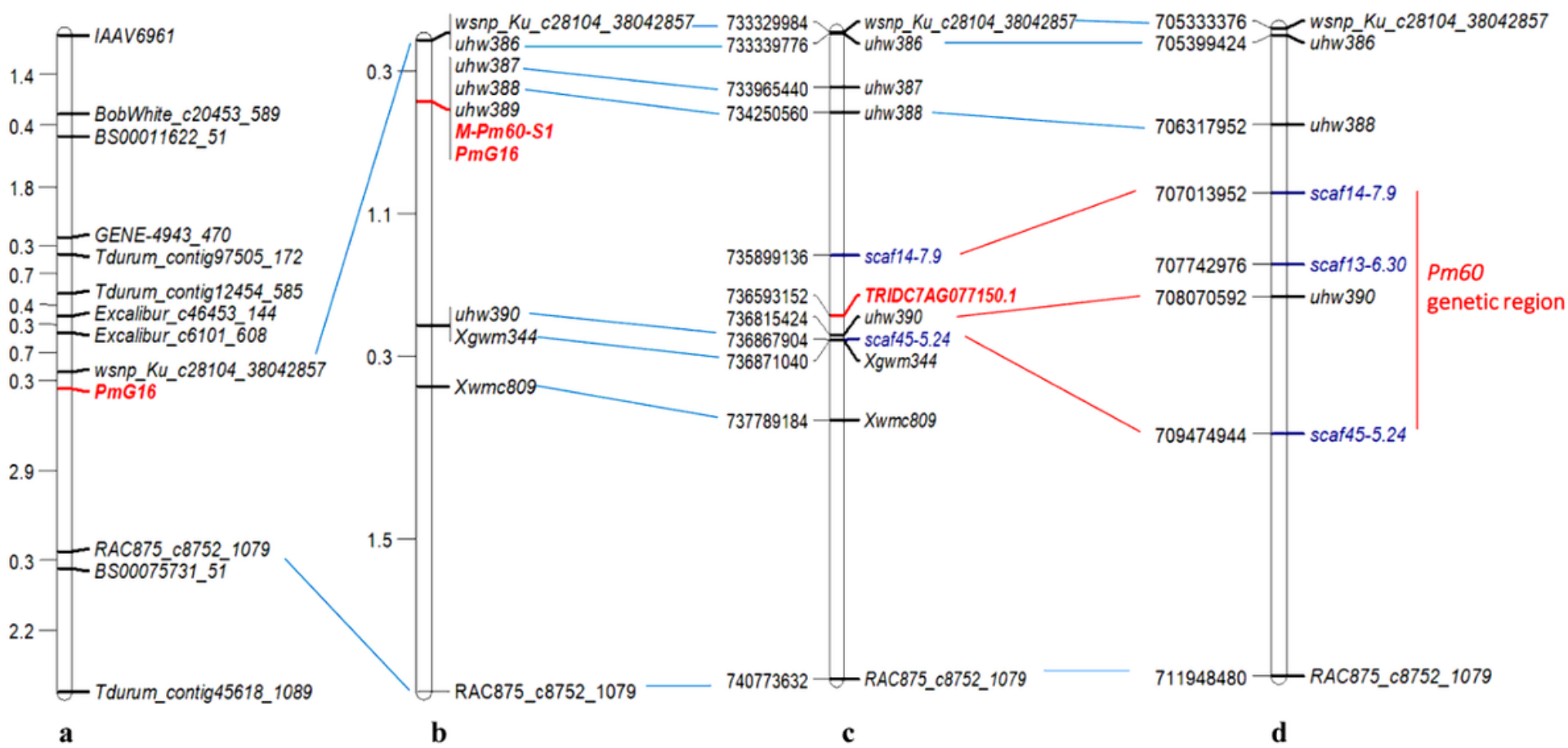

Figure 2

Genetic and physical maps of wheat 7AL containing PmG16 gene region. a Genetic map of the PmG16 gene region based on 12 SNPs from 15K SNP array. b Further dissection of the PmG16 gene region based on 2 SNP and 8 SSR markers. c Anchoring of the sub-genetic map of the PmG16 locus and the Pm60 flanking markers to the reference genome WEW Zavitan_v.2.0. d Anchoring of the sub-genetic map of the PmG16 locus and flanking markers of Pm60 to the reference genome T. urartu G1812. The flanking markers of Pm60: scaf14-7.9 and scaf45-5.24; Co-segregating marker of Pm60: scaf13-6.30. The markers are shown on the right with genetic distance or physical position on the left.

a

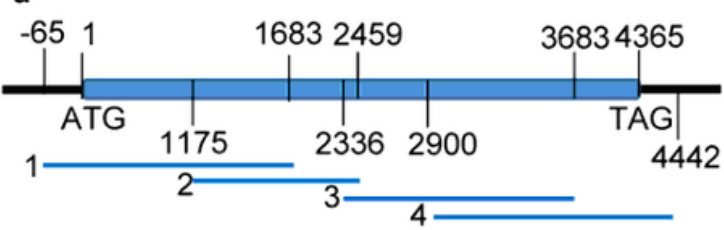

b

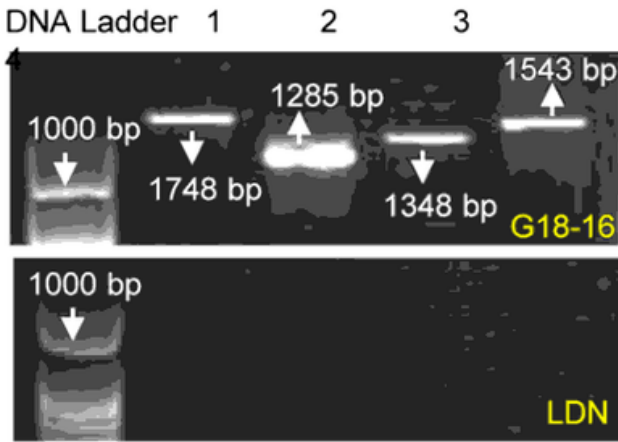

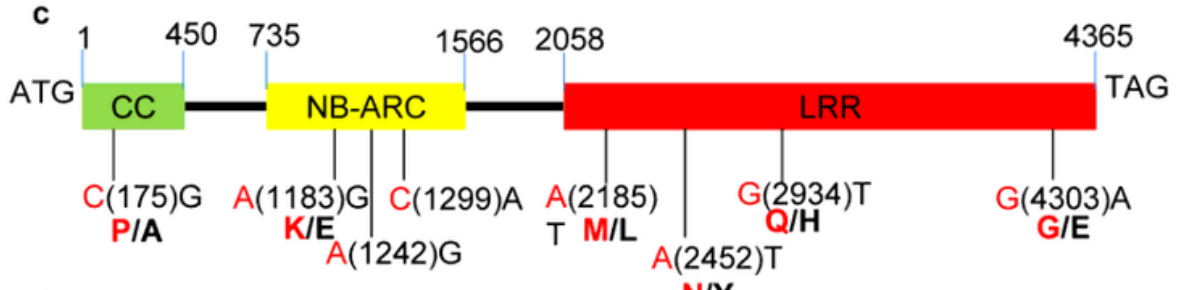

d

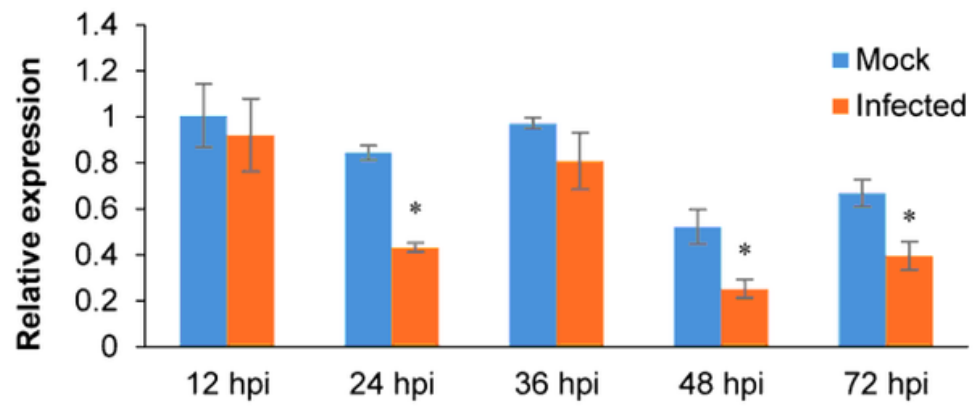




\section{Figure 3}

Schematic representation of the homologous cloning approach, conserved domains, SNPs relative to TuPm60 and expression patterns of TdPm60. a Locations of primers used for cloning and sequencing of TdPm60 from WEW by four overlapped PCR fragments (1-4). b PCR products of fragments 1-4 amplified from G18-16 and LDN. c Structures of TdPm60 conserved domains and differences from TuPm60 in nucleotides and amino acid sequences: TdPm60 (red font) and TuPm60 (bold font). d Relative expression pattern of TdPm60 after Bgt-infection in G18-16. Infected: wheat leaves infected with Bgt \#15. Mock: wheat leaves without Bgt \#15 infection.

a

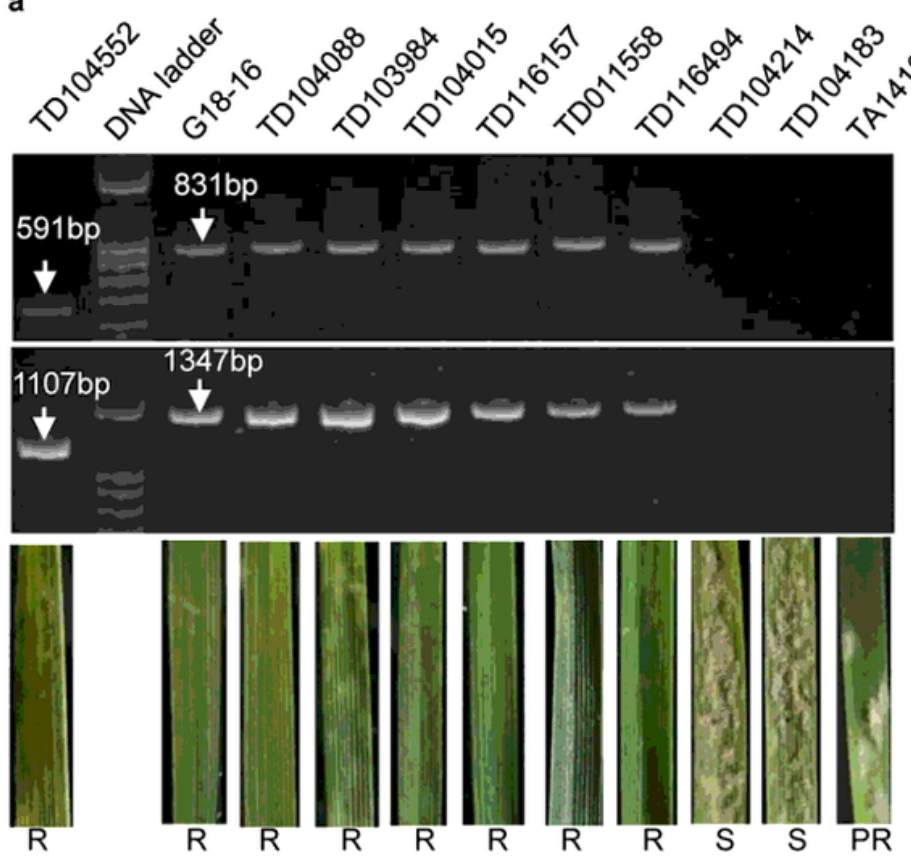

C

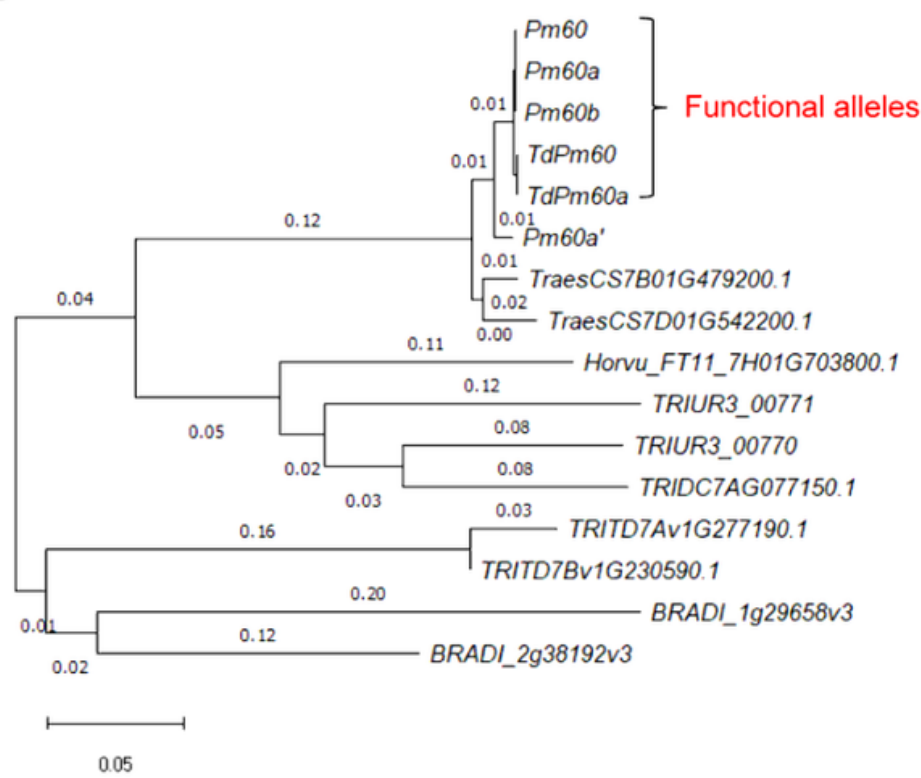

b

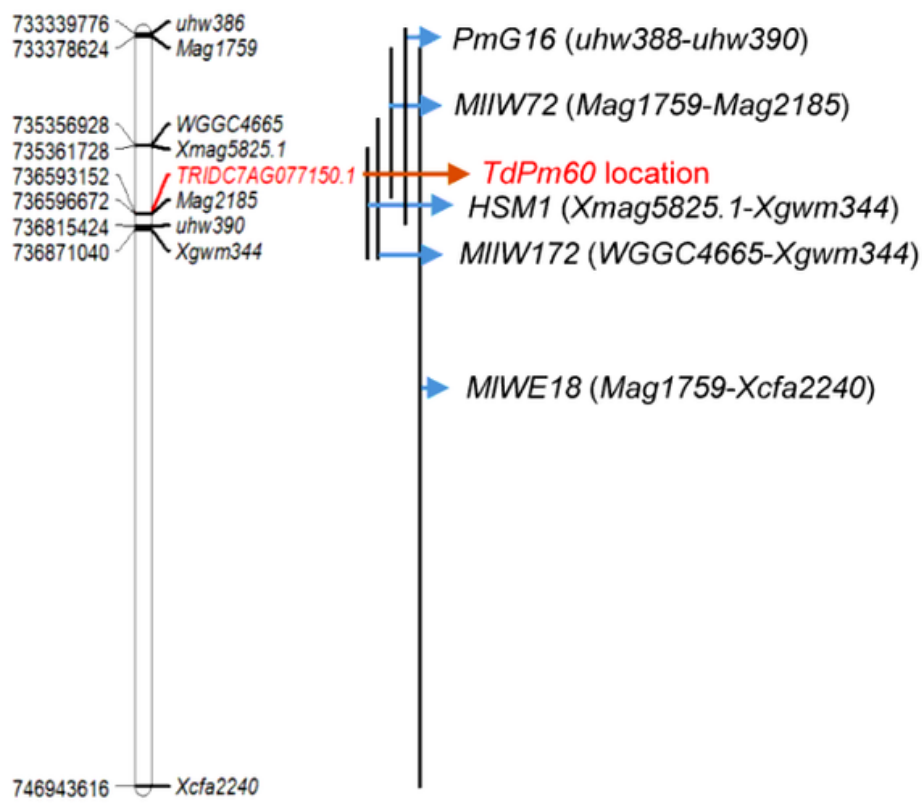

d

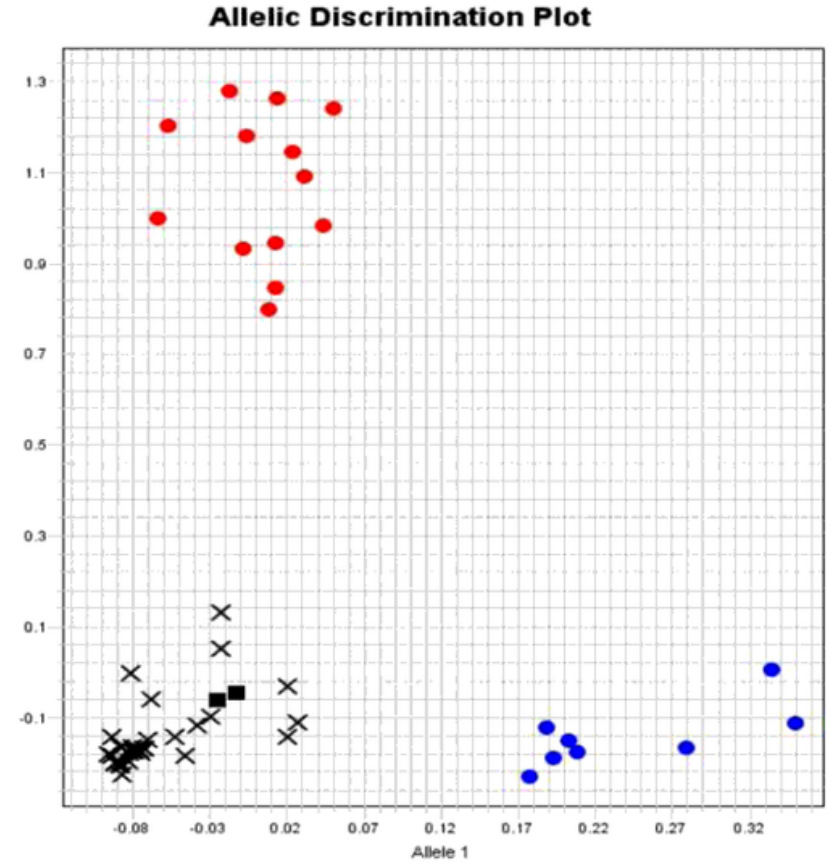

Figure 4 
TdPm60 locus analysis and functional Pm60 KASP marker development. a PCR amplification using FMMs M-Pm60-S1 and M-Pm60-S3 in the respective accession of WEW accessions at 7 dpi with Bgt \#15. $\mathrm{R}$ (resistant), Partial resistant (PR), S (susceptible) to Bgt \#15. b Anchoring of the flanking markers of MIIW72, MIIW172, PmG16 and SHM1 to the reference genome WEW Zavitan_v.2.0. c Phylogeny of Pm60 alleles and homologs from cereal species. The genetic distance is above the branch and hidden if < $0.002 \%$. d Genotyping of different wheat accessions by the functional KASP marker M-Pm60-S4. Red circle: Functional Pm60 allele; Blue circle: nonfunctional Pm60 allele; Black square: Water (negative control); Black cross: Null Pm60 allele. 


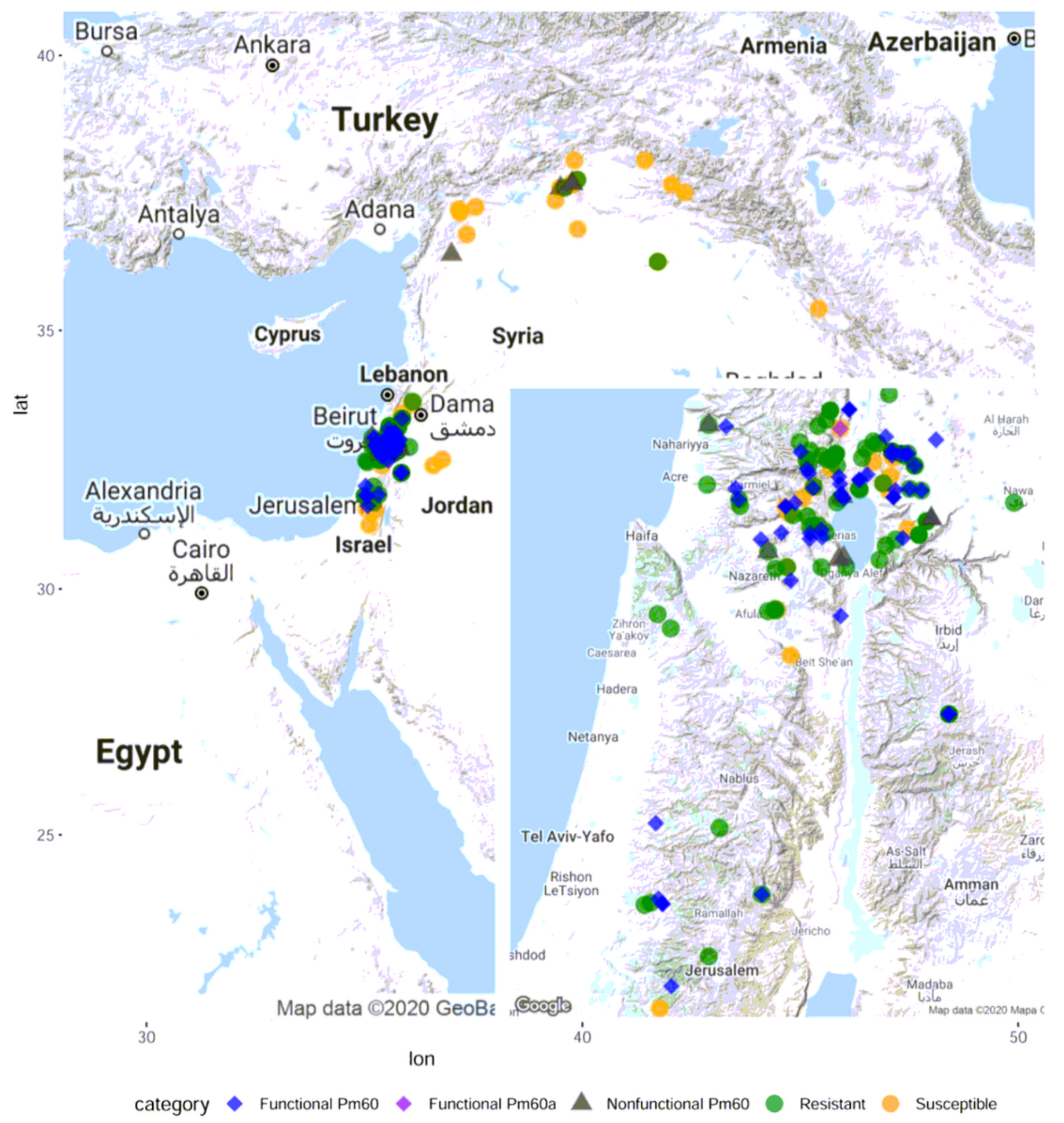

\section{Figure 5}

The Geographic distribution of the TdPm60 and TdPm60a. Blue diamond-TdPm60; Purple diamondTdPm60a; Grey triangles-nonfunctional TdPm60 alleles/homologs; Green circles-resistant accessions without TdPm60; Yellow circles-susceptible accessions without TdPm60. The resistance phenotype was checked by infection with Bgt \#15. Note: The designations employed and the presentation of the material on this map do not imply the expression of any opinion whatsoever on the part of Research Square 
concerning the legal status of any country, territory, city or area or of its authorities, or concerning the delimitation of its frontiers or boundaries. This map has been provided by the authors.

a

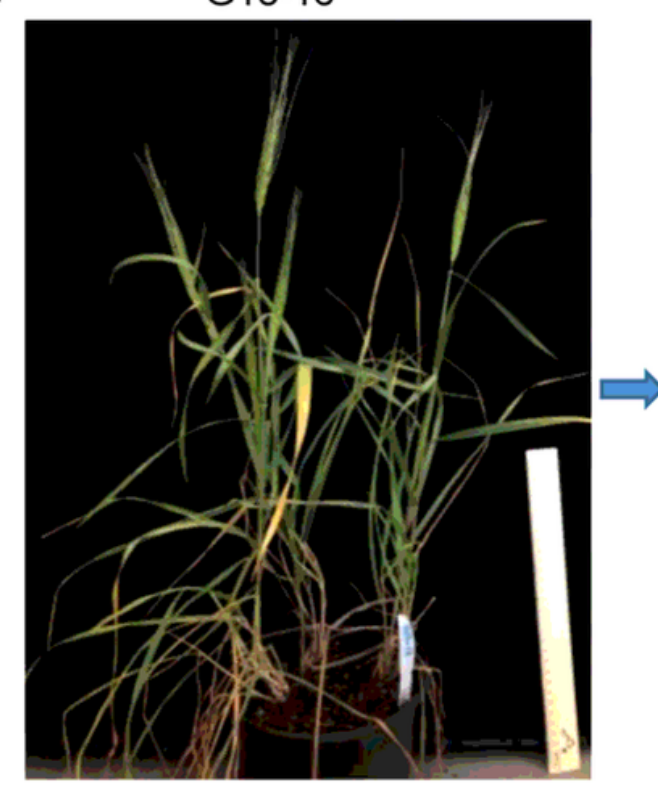

LDN

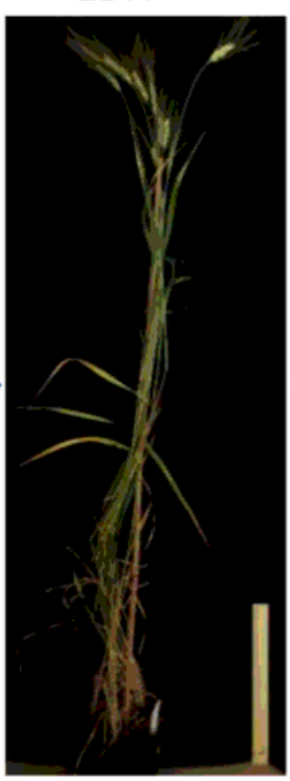

Ruta

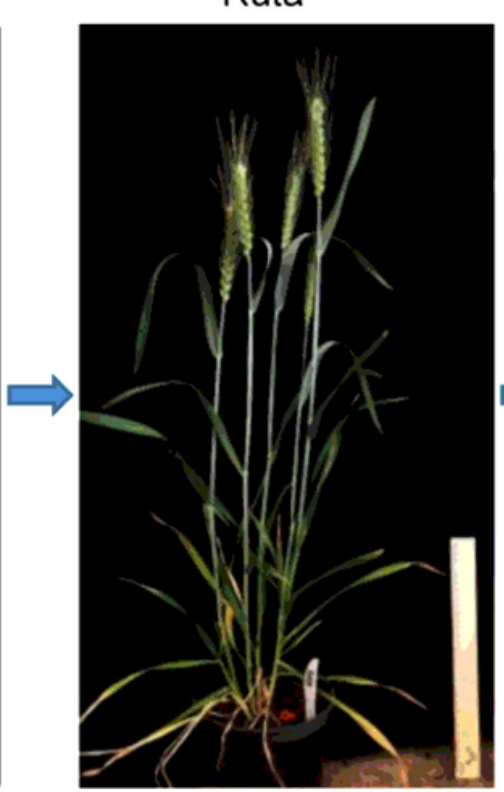

BC12-15-4
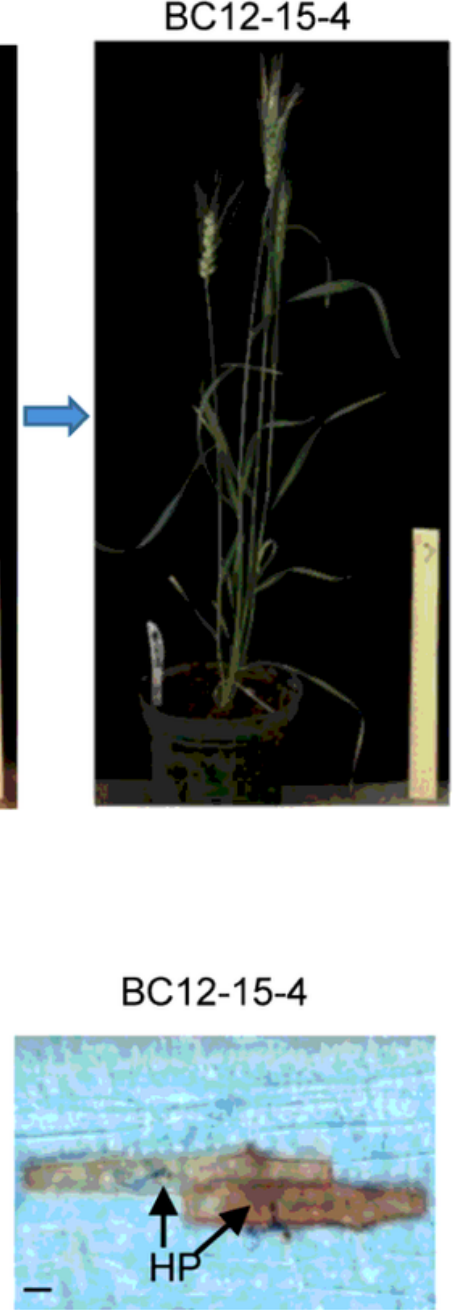

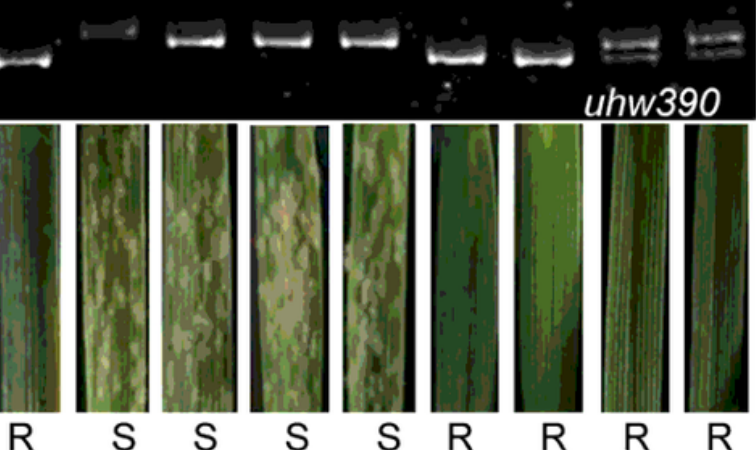

M-Pm60-S1

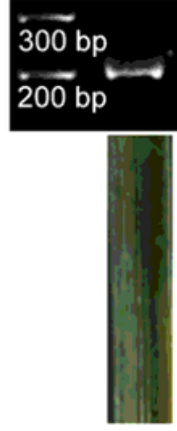

$\mathrm{R}$
S uhw390

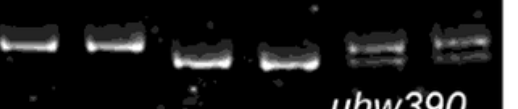

Ruta

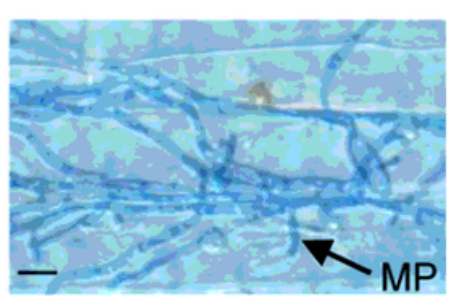

C

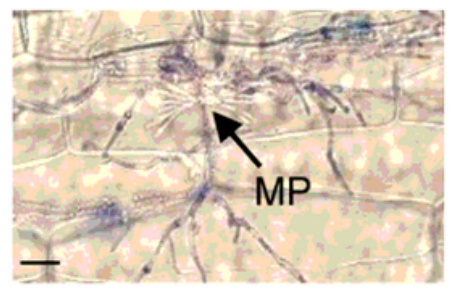

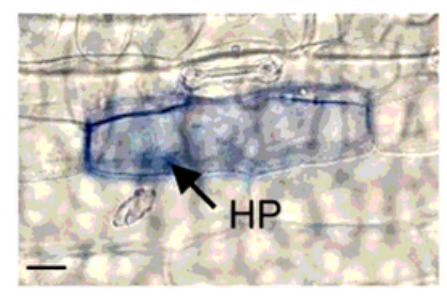

Figure 6

Development of bread wheat introgression lines. a Durum LDN as a bridge for transferring PmG16 into bread wheat Ruta. G18-16, LDN, Ruta and IL BC12-15-4 plants were grown to maturity under greenhouse conditions. Bar=32cm (Ruler). b Agarose gel electrophoresis of PCR products amplified by M-Pm60-S1 and uhw390 from G18-16, LDN, Ruta and the ILs (BC12-2-2, BC12-2-4, BC11-15-2, BC12-15-4, BC12-9-2, BC12-9-3) and their response to infection with Bgt \#15 at 7 dpi (lower row; R, resistant; S, susceptible). C DAB (upper panel) and Trypan blue (lower panel) staining of leaves infected with Bgt \#15 at 2 dpi, indicates the accumulation of ROS (reddish-brown coloration) and plant cell death (blue coloration) in wheat leaves. MP, mature haustorium; HP, haustorial primordia. Bars $=20 \mu \mathrm{m}$. 


\section{Supplementary Files}

This is a list of supplementary files associated with this preprint. Click to download.

- Fig.S1S5.pdf

- TableS1S10.xIsx 